\title{
TWO-SCALE HOMOGENIZATION FOR A MODEL IN STRAIN GRADIENT PLASTICITY
}

\author{
Alessandro Giacomini ${ }^{1}$ And Alessandro Musesti $^{2}$
}

\begin{abstract}
Using the tool of two-scale convergence, we provide a rigorous mathematical setting for the homogenization result obtained by Fleck and Willis [J. Mech. Phys. Solids 52 (2004) 18551888] concerning the effective plastic behaviour of a strain gradient composite material. Moreover, moving from deformation theory to flow theory, we prove a convergence result for the homogenization of quasistatic evolutions in the presence of isotropic linear hardening.
\end{abstract}

Mathematics Subject Classification. 74C05, 74G65, 74Q05, 35B27, 49J45.

Received October 12, 2009. Revised April 2, 2010 and May 5, 2010.

Published online October 28, 2010.

\section{INTRODUCTION}

Strain gradient plasticity models have been deeply studied in recent years in order to understand size effects taking place in ductile metals (see $[7,8,12,13]$ and references therein). The gradient of the plastic strain is connected with the density of geometrically necessary dislocations inside the body (see [2]) and its inclusion in the model aims at capturing their interactions. However, the way in which such a term affects the equations of the model is still suggested by phenomenological considerations, although in agreement with the general principles of thermodynamics (see [12,13]). Strain gradient terms may play both a dissipative and an energetic role; if the configuration of an elastoplastic body $\Omega \subseteq \mathbb{R}^{N}$ subject to small displacements $u: \Omega \rightarrow \mathbb{R}^{N}$ entails a plastic strain $p: \Omega \rightarrow \mathrm{M}_{D}^{N}$ (here $\mathrm{M}_{D}^{N}$ stands for the space of symmetric deviatoric matrices, see Sect. 2), an overall plastic strain measure usually employed to compute the dissipation during an evolution is given by the quantity

$$
\sqrt{|\dot{p}|^{2}+\ell^{2}|\nabla \dot{p}|^{2}}
$$

Here $\ell$ is a dissipative length-scale, which has the dimension of a length and the order of magnitude of the distance at which interactions between dislocations take place. In particular, for polycrystals, $\ell$ is comparable with the size of the grains of the material.

In 2004 Fleck and Willis [9] studied the behaviour of composite materials with highly oscillating elastic and plastic moduli, whose response in the homogenization limit does not involve gradient terms. More precisely,

\footnotetext{
Keywords and phrases. Strain gradient plasticity, periodic homogenization, two-scale convergence, quasistatic evolutions.

1 Dipartimento di Matematica, Facoltà di Ingegneria, Università degli Studi di Brescia, Via Valotti 9, 25133 Brescia, Italy. alessandro.giacomini@ing.unibs.it

2 Dipartimento di Matematica e Fisica "Niccolò Tartaglia", Università Cattolica del Sacro Cuore, Via dei Musei 41, 25121 Brescia, Italy. alessandro.musesti@unicatt.it
} 
they considered a strain gradient deformation theory whose associated energy is given by

$$
\mathcal{E}(u, p)=\frac{1}{2} \int_{\Omega} \mathbb{C}(x)(E u-p):(E u-p) \mathrm{d} x+\int_{\Omega} b(x)\left[|p|^{2}+\ell^{2}|\nabla p|^{2}\right] \mathrm{d} x,
$$

where the elastic tensor $\mathbb{C}$ and the yielding function $b$ highly oscillate in space, and $E u$ denotes the symmetrized gradient of $u$. Since the interactions modeled by strain gradients tend to vanish in the homogenization limit, Fleck and Willis focused on the problem of finding suitable bounds for the effective energy (independent of $\nabla p$ )

$$
\mathcal{E}^{\mathrm{eff}}(u, p)=\int_{\Omega} F^{\mathrm{eff}}(E u(x), p(x)) \mathrm{d} x
$$

governing the behaviour of the homogenized body. Here the effective energy density $F^{\mathrm{eff}}(\bar{A}, \bar{p})$ is provided by minimizing the energy (1.1) on a representative volume element, among displacement fields $u$ satisfying the linear boundary condition $u=\bar{A} \cdot x$ and plastic strains $p$ with mean given by $\bar{p}$. In the particular case when $\mathbb{C}$ is constant and only the yielding function $b$ oscillates, the effective energy density becomes

$$
F^{\mathrm{eff}}(\bar{A}, \bar{p})=\frac{1}{2} \mathbb{C}(\bar{A}-\bar{p}):(\bar{A}-\bar{p})+V^{\mathrm{eff}}(\bar{p}),
$$

where the elastic part is clearly identified, but the effective plastic potential $V^{\text {eff }}$ depends also on the elastic properties of $\Omega$. Indeed, its expression involves an operator $\Gamma$ (introduced by Willis in [25]) associated to an elasticity problem which depends on $\mathbb{C}$ (see Thm. 4.7).

The first aim of our paper is to provide a rigorous mathematical framework in order to establish (1.2) in the case when $\mathbb{C}$ and $b$ oscillate in a periodic way. We consider energies of the form

$$
\mathcal{E}_{\varepsilon}(u, p)=\frac{1}{2} \int_{\Omega} \mathbb{C}\left(\frac{x}{\varepsilon}\right)(E u-p):(E u-p) \mathrm{d} x+\int_{\Omega} b\left(\frac{x}{\varepsilon}\right)\left[|p|^{2}+\varepsilon^{2} \ell^{2}|\nabla p|^{2}\right] \mathrm{d} x,
$$

defined on $H^{1}\left(\Omega ; \mathbb{R}^{N}\right) \times H^{1}\left(\Omega ; \mathrm{M}_{D}^{N}\right)$, where $\mathbb{C}$ and $b$ are periodic and satisfy suitable coercivity assumptions. The plastic and elastic moduli oscillate on a scale $\varepsilon$; accordingly, the dissipative length-scale is given by $\varepsilon \ell$, with $\ell>0$.

We study the asymptotic behaviour of $\mathcal{E}_{\varepsilon}$ as $\varepsilon \rightarrow 0$ in the framework of two-scale convergence. This remarkable notion (see Sect. 3 for the precise definition and the main properties) has been introduced by Nguetseng [19] and Allaire [1] in order to study periodic homogenization in linearized elasticity. Let us consider the unit cube

$$
Y:=\left[-\frac{1}{2}, \frac{1}{2}\left[^{N}\right.\right.
$$

For a family of functions $\left(v_{\varepsilon}\right)_{\varepsilon>0}$ uniformly bounded in $L^{p}(\Omega)$, the two-scale weak limit of $v_{\varepsilon}$ is given by $V \in L^{p}(\Omega \times Y)$ such that

$$
\lim _{\varepsilon \rightarrow 0} \int_{\Omega} v_{\varepsilon}(x) \psi\left(x, \frac{x}{\varepsilon}\right) \mathrm{d} x=\int_{\Omega \times Y} V(x, y) \psi(x, y) \mathrm{d} x \mathrm{~d} y
$$

for every smooth function $\psi(x, y)$ defined on $\Omega \times Y$ and periodic in $y$ (see Def. 3.1). Noticeably, a microstructural variable $y$ appears in order to keep track of the oscillations of the functions of the family. In Section 4 we will prove that the asymptotic behaviour of (1.4) along a family $\left(u_{\varepsilon}, p_{\varepsilon}\right)_{\varepsilon>0}$ can be inferred from the two-scale energy

$$
\mathcal{E}(u, U, P)=\frac{1}{2} \int_{\Omega \times Y} \mathbb{C}(y)\left(E u+E_{y} U-P\right):\left(E u+E_{y} U-P\right) \mathrm{d} x \mathrm{~d} y+\int_{\Omega \times Y} b(y)\left[|P|^{2}+\ell^{2}\left|\nabla_{y} P\right|^{2}\right] \mathrm{d} x \mathrm{~d} y,
$$


where $U \in L^{2}\left(\Omega ; H_{\mathrm{per}, 0}^{1}\left(Y ; \mathbb{R}^{N}\right)\right)$, periodic and with null average in $y$, is connected with the two-scale weak limit of $E u_{\varepsilon}$ (see Prop. 3.3), while $P \in L^{2}\left(\Omega ; H_{\mathrm{per}}^{1}\left(Y ; \mathrm{M}_{D}^{N}\right)\right)$, periodic in $y$, is associated with the two-scale weak limit of $p_{\varepsilon}$ and $\varepsilon \nabla p_{\varepsilon}$ (see Thm. 3.5). By employing (1.5), we will show (Thms. 4.3 and 4.5) that the configurations $\left(u_{\varepsilon}, p_{\varepsilon}\right)$ which minimize (under suitable boundary conditions for the displacement)

$$
(u, p) \mapsto \mathcal{E}_{\varepsilon}(u, p)-\int_{\Omega} f \cdot u \mathrm{~d} x,
$$

where $f \in L^{2}\left(\Omega ; \mathbb{R}^{N}\right)$ stands for the density per unit volume of external body forces, converge as $\varepsilon \rightarrow 0$ in the weak topology of $H^{1}\left(\Omega ; \mathbb{R}^{N}\right) \times L^{2}\left(\Omega ; \mathrm{M}_{D}^{N}\right)$ to the minimizer of

$$
(u, p) \mapsto \mathcal{E}^{\mathrm{eff}}(u, p)-\int_{\Omega} f \cdot u \mathrm{~d} x
$$

where $\mathcal{E}^{\text {eff }}$ is of the form (1.2). Moreover, concerning the effective energy density we obtain the formula

$$
\begin{aligned}
F^{\mathrm{eff}}(\bar{A}, \bar{p}):=\min & \left\{\frac{1}{2} \int_{Y} \mathbb{C}(y)\left[\bar{A}+E_{y} U-P\right]:\left[\bar{A}+E_{y} U-P\right] \mathrm{d} y\right. \\
& \left.+\int_{Y} b(y)\left[|P|^{2}+\ell^{2}\left|\nabla_{y} P\right|^{2}\right] \mathrm{d} y:(U, P) \in H_{\mathrm{per}, 0}^{1}\left(Y ; \mathbb{R}^{N}\right) \times H_{\mathrm{per}}^{1}\left(Y ; \mathrm{M}_{D}^{N}\right), \int_{Y} P(y) \mathrm{d} y=\bar{p}\right\}
\end{aligned}
$$

in which the representative volume element is precisely the unit cell $Y$. In the case when the elasticity tensor is constant and oscillations do occur only in the yielding function, we obtain a characterization of Willis' operator $\Gamma$ in terms of the function $U$ appearing in (1.5) (see Def. 4.6 and Thm. 4.7).

The key tool in investigating the asymptotic behaviour as $\varepsilon \rightarrow 0$ of the energies (1.4) is Theorem 3.5, where an asymptotic and approximation result in a two-scale sense concerning functions $v_{\varepsilon}$ bounded in $L^{2}(\Omega)$ with $\varepsilon \nabla v_{\varepsilon}$ bounded in $L^{2}\left(\Omega ; \mathbb{R}^{N}\right)$ is given.

In Section 5 we move from deformation theory to flow theory, considering quasistatic evolutions for the model associated to (1.1) in the presence of isotropic linear hardening. Setting again the problem in the case of periodic oscillations for the elastic and plastic moduli, we study the asymptotic behaviour of quasistatic evolutions with vanishing strain gradient effects, employing the energetic approach to evolutions for rate independent systems introduced by Mielke and his school (see [16] and references therein). In this framework, the analysis of the deformation theory can be considered as a preliminary step for the study of the corresponding flow theory. We show in Theorem 5.8 that the homogenization of quasistatic evolutions can be understood moving to a two-scale setting and considering a suitable notion of quasistatic evolution within this context (see Def. 5.4): even if strain gradient effects tend to vanish, the model turns out to be of strain gradient type with respect to the microstructural variable $y$. The passage to a single scale setting seems to lead to an evolution which cannot be described in terms of standard plasticity models associated to the effective energy (1.2) (see Rem. 5.9).

The paper is organized as follows. In Section 2 we state the notation employed throughout the paper, while in Section 3 we recall the definition and the basic properties of two-scale convergence which will be essential in Section 4 when dealing with the two-scale approach to the homogenization procedure of Fleck and Willis. Finally, Section 5 is devoted to the homogenization of strain gradient quasistatic evolutions with isotropic linear hardening.

\section{Notation AND PRELIMINARIES}

In this section we introduce the notation and recall some basic definitions concerning the functional spaces employed in the rest of the paper. In the following, $B_{r}(x)$ will denote the open ball of center $x \in \mathbb{R}^{N}$ and radius $r>0$. If $E \subset \mathbb{R}^{N}$, we will denote its volume by $|E|$, and $1_{E}$ will stand for its characteristic function, i.e., $1_{E}(x)=1$ if $x \in E$ and $1_{E}(x)=0$ if $x \notin E$. 
Matrices. On the space of $N \times N$ matrices $A=\left(a_{i j}\right)$ with $a_{i j} \in \mathbb{R}$ we will consider the scalar product

$$
A: B:=\sum_{i, j} a_{i j} b_{i j}
$$

The associated norm of $A$ is denoted by $|A|$.

We will denote by $\mathrm{M}_{\mathrm{sym}}^{N}$ the subspace of symmetric matrices, and by $\mathrm{M}_{D}^{N}$ the subspace of $\mathrm{M}_{\mathrm{sym}}^{N}$ of deviatoric matrices $A$, that is such that $\operatorname{tr} A:=\sum_{i} a_{i i}=0$.

The symmetrized gradient of a $\mathbb{R}^{N}$-valued function $u(x)$ is defined as

$$
E u:=\frac{\nabla u+\nabla u^{T}}{2},
$$

where $(\nabla u)_{i j}=\frac{\partial u_{i}}{\partial x_{j}}$ is the gradient of $u$ and $\nabla u^{T}$ denotes its transpose.

The gradient of a matrix-valued function $A(x)=\left(a_{i j}(x)\right)$ is defined as the third-order tensor

$$
(\nabla A)_{i j k}:=\frac{\partial a_{i j}}{\partial x_{k}}
$$

We will consider on the space of third order tensors $\mathbb{A}=\left(a_{i j k}\right)$ the norm

$$
|\mathbb{A}|:=\sqrt{\sum_{i, j, k} a_{i j k}^{2}} .
$$

We say that $\mathbb{A}=\left(a_{i j k}\right)$ is symmetric-deviatoric in its first two subscripts if

$$
a_{i j k}=a_{j i k} \quad \text { and } \quad \sum_{p} a_{p p k}=0
$$

and we write $\mathbb{A} \in \mathbb{M}_{D}^{N}$.

Functional spaces. Throughout the paper, given $E \subseteq \mathbb{R}^{N}$ measurable and $X$ a finite dimensional normed space, $L^{p}(E ; X)$ with $p \in\left[1 ;+\infty\left[\right.\right.$ will stand for the space of $p$-summable functions with values in $X . L^{\infty}(E ; X)$ will denote the space of essentially bounded maps from $E$ to $X$, and $\|\cdot\|_{\infty}$ will be the associated sup-norm. Given $A \subseteq \mathbb{R}^{N}$ open, $W^{1, p}(A ; X)$ will denote the usual Sobolev space of functions in $L^{p}(A ; X)$ whose distributional derivatives are $p$-summable, and $W_{0}^{1, p}(A ; X)$ will denote the subspace of functions vanishing at the boundary. For $p=2$ we write $H^{1}(A ; X)$ and $H_{0}^{1}(A ; X)$ in place of $W^{1,2}(A ; X)$ and $W_{0}^{1,2}(A ; X)$ respectively. If $X=\mathbb{R}$, as usual we will write $L^{p}(E), W^{1, p}(A)$ and $W_{0}^{1, p}(A)$.

Let us set

$$
Y:=\left[-\frac{1}{2}, \frac{1}{2}\left[^{N} .\right.\right.
$$

We will refer to $Y$ as the unit cell, and write $W^{1, p}(Y ; X)$ in place of $W^{1, p}(\operatorname{int}(Y) ; X)$. Moreover we set

and

$$
\begin{gathered}
W_{\text {per }}^{1, p}(Y ; X):=\left\{u \in W^{1, p}(Y ; X): u \text { admits a } Y \text {-periodic extension to } \mathbb{R}^{N}\right\}, \\
H_{\text {per }}^{1}(Y ; X):=W_{\text {per }}^{1,2}(Y ; X),
\end{gathered}
$$

$$
\begin{gathered}
W_{\text {per }, 0}^{1, p}(Y ; X):=\left\{u \in W_{\text {per }}^{1, p}(Y ; X): \int_{Y} u \mathrm{~d} y=0\right\}, \\
H_{\text {per }, 0}^{1}(Y ; X):=W_{\text {per }, 0}^{1,2}(Y ; X) .
\end{gathered}
$$


Notice that $u \in W_{\text {per }}^{1, p}(Y ; X)$ can be characterized in terms of traces on the faces of $Y$. For $i=1, \ldots, N$ set

$$
\partial_{i}^{ \pm} Y:=\left\{y \in \bar{Y}: y_{i}= \pm \frac{1}{2}\right\}
$$

and let $\gamma_{i}^{ \pm}$denote the trace operator from $W^{1, p}(Y ; X)$ to $L^{p}\left(\partial_{i}^{ \pm} Y ; X\right)$. The spaces $L^{p}\left(\partial_{i}^{ \pm} Y ; X\right)$ can be identified naturally with $L^{p}(]-1 / 2,1 / 2\left[^{N-1} ; X\right)$ : it turns out easily that $u \in W_{\text {per }}^{1, p}(Y ; X)$ if and only if $u \in W^{1, p}(Y ; X)$ and $\gamma_{i}^{+}(u)=\gamma_{i}^{-}(u)$ for every $i=1, \ldots, N$.

Korn's inequality. Dealing with linearized elasticity, we will employ several times the following inequality due to Korn.

Theorem 2.1. Let $\Omega \subseteq \mathbb{R}^{N}$ be open. Then there exists $C>0$ such that for every $u \in H_{0}^{1}\left(\Omega ; \mathbb{R}^{N}\right)$

$$
\|\nabla u\|_{L^{2}\left(\Omega ; \mathbb{R}^{N \times N}\right)} \leq C\|E u\|_{L^{2}\left(\Omega ; \mathrm{M}_{\text {sym }}^{N}\right)}
$$

where Eu denotes the symmetrized gradient of $u$.

The proof easily follows performing integration by parts (see e.g. [6], Sect. 7.3, Rem. 15) or by extending $u$ to $\mathbb{R}^{N}$ and applying Plancherel's formula for Fourier transforms. The same inequality holds also for functions in $H_{\mathrm{per}, 0}^{1}\left(Y ; \mathbb{R}^{N}\right), Y$ being the unit cell $(2.1)$, the proof following by expansion in Fourier series.

\section{TWO-SCALE CONVERGENCE}

Introduced in the seminal papers of Nguetseng [19] and Allaire [1] about twenty years ago, two-scale convergence is nowadays a pretty well-known notion. Dealing with periodic functions with a precise scale parameter, it revealed as a powerful tool in performing periodic homogenization.

In this section we recall some basic facts concerning two-scale convergence, and we prove an approximation result (Thm. 3.5) which will be essential for our analysis of the homogenization procedure in strain gradient plasticity proposed by Fleck and Willis [9].

Let $\Omega$ be an open bounded subset of $\mathbb{R}^{N}$ with $|\partial \Omega|=0$, and let $\left.p \in\right] 1,+\infty[$. The definition of two-scale convergence for functions in $L^{p}$ is based on a duality argument employing finely oscillating test functions. In view of our applications, we restrict to the case of bounded sequences.

Definition 3.1 (two-scale convergence). Let $\left(u_{\varepsilon}\right)_{\varepsilon>0}$ be a bounded family in $L^{p}(\Omega)$. We say that $u_{\varepsilon}$ converges two-scale weakly to $U \in L^{p}(\Omega \times Y)$ for $\varepsilon \rightarrow 0$, and write

$$
u_{\varepsilon} \stackrel{w-2}{\longrightarrow} U \quad \text { two-scale weakly in } L^{p}(\Omega \times Y)
$$

provided that

$$
\lim _{\varepsilon \rightarrow 0} \int_{\Omega} u_{\varepsilon}(x) \psi\left(x, \frac{x}{\varepsilon}\right) \mathrm{d} x=\int_{\Omega \times Y} U(x, y) \psi(x, y) \mathrm{d} x \mathrm{~d} y
$$

for every $\psi \in L^{p^{\prime}}\left(\Omega ; C_{\mathrm{per}}^{0}(\bar{Y})\right)\left(p^{\prime}:=p / p-1\right)$.

We say that $u_{\varepsilon}$ converges two-scale strongly to $U$ for $\varepsilon \rightarrow 0$, and write

$$
u_{\varepsilon} \stackrel{s-2}{\rightarrow} U \quad \text { two-scale strongly in } L^{p}(\Omega \times Y)
$$

if $u_{\varepsilon} \stackrel{w-2}{\longrightarrow} U$ two-scale weakly in $L^{p}(\Omega \times Y)$ and $\lim _{\varepsilon \rightarrow 0}\left\|u_{\varepsilon}\right\|_{L^{p}(\Omega)}=\|U\|_{L^{p}(\Omega \times Y)}$. 
In view of the boundedness of $\left(u_{\varepsilon}\right)_{\varepsilon>0}$, this definition turns out to be equivalent to the original one which employs smooth test functions (see [14], Prop. 1). Notice that taking $\psi$ independent of $y$ in (3.1), it follows that

$$
u_{\varepsilon}(x) \rightarrow u(x):=\int_{Y} U(x, y) \mathrm{d} y \quad \text { weakly in } L^{p}(\Omega),
$$

i.e., the average with respect to $y$ of the two-scale weak limit $U$ yields the usual weak limit of $u_{\varepsilon}$ in $L^{p}(\Omega)$.

Two-scale weak/strong convergence can be reinterpreted as usual weak/strong convergence in the doublevariable space $L^{p}(\Omega \times Y)$ provided that we employ the notion of periodic unfolding operator firstly introduced in [3]. We refer the reader to [4] for a survey of this approach and for applications to several periodic homogenization problems. We will follow the interpretation of the method proposed by Mielke and Timofte in [18] and we will employ their notation.

Performing for $\varepsilon>0$ and $x \in \mathbb{R}^{N}$ the unique decomposition

$$
x=\mathcal{N}_{\varepsilon}(x)+\varepsilon \mathcal{R}_{\varepsilon}(x), \quad \frac{1}{\varepsilon} \mathcal{N}_{\varepsilon}(x) \in \mathbb{Z}^{N}, \quad \mathcal{R}_{\varepsilon}(x) \in Y,
$$

where $Y$ is the unit cell defined in $(2.1)$, let $\mathcal{D}_{\varepsilon}: \mathbb{R}^{N} \rightarrow \mathbb{R}^{N} \times Y$ and $\mathcal{S}_{\varepsilon}: \mathbb{R}^{N} \times Y \rightarrow \mathbb{R}^{N}$ be defined as

$$
\mathcal{D}_{\varepsilon}(x):=\left(\mathcal{N}_{\varepsilon}(x), \mathcal{R}_{\varepsilon}(x)\right) \quad \text { and } \quad \mathcal{S}_{\varepsilon}(x, y):=\mathcal{N}_{\varepsilon}(x)+\varepsilon y .
$$

Let us denote by $v 1_{\Omega}$ the extension of $v \in L^{p}(\Omega)$ to all $\mathbb{R}^{N}$ with value 0 outside $\Omega$. The periodic unfolding operator $\mathcal{T}_{\varepsilon}: L^{p}(\Omega) \rightarrow L^{p}\left(\mathbb{R}^{N} \times Y\right)$ is the isometry defined as

$$
\mathcal{T}_{\varepsilon}(v):=\left(v 1_{\Omega}\right) \circ \mathcal{S}_{\varepsilon}
$$

Let $\left(u_{\varepsilon}\right)_{\varepsilon>0}$ be a family bounded in $L^{p}(\Omega)$, and $U \in L^{p}(\Omega \times Y)$. Let us extend $U$ to $\mathbb{R}^{N} \times Y$ by setting $U=0$ outside $\Omega \times Y$. Then (see [4], Prop. 2.14 and [18], Prop. 2.5)

$$
u_{\varepsilon} \stackrel{w-2}{=} U \quad \text { two-scale weakly in } L^{p}(\Omega \times Y)
$$

if and only if

$$
\mathcal{T}_{\varepsilon} u_{\varepsilon} \rightarrow U \quad \text { weakly in } L^{p}\left(\mathbb{R}^{N} \times Y\right)
$$

Similarly

if and only if

$$
u_{\varepsilon} \stackrel{s-2}{\rightarrow} U \quad \text { two-scale strongly in } L^{p}(\Omega \times Y)
$$

$$
\mathcal{T}_{\varepsilon} u_{\varepsilon} \rightarrow U \quad \text { strongly in } L^{p}\left(\mathbb{R}^{N} \times Y\right) .
$$

In order to approximate functions in $L^{p}(\Omega \times Y)$ by means of functions in $L^{p}(\Omega)$ in the two-scale sense, it is useful to have an operation dual with respect to the periodic unfolding. This was introduced under the name of averaging operator in [3]. Following the reinterpretation of [18], for any $U \in L^{p}\left(\mathbb{R}^{N} \times Y\right)$ let

$$
\mathcal{P}_{\varepsilon}(U)(x, y):=\frac{1}{\varepsilon^{N}} \int_{\mathcal{N}_{\varepsilon}(x)+\varepsilon Y} U(\xi, y) \mathrm{d} \xi
$$

be the projection of $U$ on the space of piecewise constant functions with respect to $x \in \mathbb{R}^{N}$. Then the averaging operator $\mathcal{F}_{\varepsilon}: L^{p}(\Omega \times Y) \rightarrow L^{p}(\Omega)$ is given by

$$
\mathcal{F}_{\varepsilon}(U):=\left.\left(\mathcal{P}_{\varepsilon}\left(1_{[\Omega \times Y]_{\varepsilon}} U\right) \circ \mathcal{D}_{\varepsilon}\right)\right|_{\Omega},
$$


where $[\Omega \times Y]_{\varepsilon}:=\left\{(x, y) \in \mathbb{R}^{N} \times Y: \mathcal{S}_{\varepsilon}(x, y) \in \Omega\right\}$, and $U$ is extended to $\mathbb{R}^{N} \times Y$ by setting $U=0$ outside $\Omega \times Y$.

The following properties are quite easy to prove (see [18], Props. 2.4 and 2.6).

Proposition 3.2 (basic properties of two-scale convergence). Let $\left(u_{\varepsilon}\right)_{\varepsilon>0}$ be a family bounded in $L^{p}(\Omega)$, $U \in L^{p}(\Omega \times Y)$ and $\left.p \in\right] 1,+\infty[$.

(1) $\left(u_{\varepsilon}\right)_{\varepsilon>0}$ is two-scale weakly convergent in $L^{p}(\Omega \times Y)$ along a suitable sequence $\varepsilon_{n} \rightarrow 0$.

(2) $\mathcal{F}_{\varepsilon}(U) \stackrel{s-2}{\rightarrow} U$ two-scale strongly in $L^{p}(\Omega \times Y)$ as $\varepsilon \rightarrow 0$.

(3) If $u_{\varepsilon} \stackrel{w-2}{\longrightarrow} U$ two-scale weakly in $L^{p}(\Omega \times Y)$ and $v_{\varepsilon} \stackrel{s-2}{\longrightarrow} V$ two-scale strongly in $L^{p^{\prime}}(\Omega \times Y)$,

$$
\lim _{\varepsilon \rightarrow 0} \int_{\Omega} u_{\varepsilon} v_{\varepsilon} \mathrm{d} x=\int_{\Omega \times Y} U V \mathrm{~d} x \mathrm{~d} y .
$$

(4) If $u_{\varepsilon} \stackrel{s-2}{\longrightarrow} U$ two-scale strongly in $L^{p}(\Omega \times Y)$, and if $\left(m_{\varepsilon}\right)_{\varepsilon>0}$ is a bounded family in $L^{\infty}(\Omega)$ such that $\mathcal{T}_{\varepsilon}\left(m_{\varepsilon}\right) \rightarrow M$ a.e. in $\mathbb{R}^{N} \times Y$, then

$$
m_{\varepsilon} u_{\varepsilon} \stackrel{s-2}{\rightarrow} M U \quad \text { two-scale strongly in } L^{p}(\Omega \times Y) .
$$

Let us now recall the main results on the two-scale convergence of derivatives of a Sobolev function. For $u \in L^{p}(\Omega)$, we denote with the same symbol $u$ the function in $L^{p}(\Omega \times Y)$ such that $(x, y) \mapsto u(x)$. Then the following proposition holds (see e.g. [18], Prop. 2.9).

Proposition 3.3 (two-scale convergence of gradients). Let $\left(u_{\varepsilon}\right)_{\varepsilon>0}$ be a family in $\left.W^{1, p}(\Omega), p \in\right] 1,+\infty[$, such that $u_{\varepsilon} \rightarrow u$ weakly in $W^{1, p}(\Omega)$ for $\varepsilon \rightarrow 0$. Then

$$
u_{\varepsilon} \stackrel{s-2}{\rightarrow} u \quad \text { two-scale strongly in } L^{p}(\Omega \times Y)
$$

and there exists $U \in L^{p}\left(\Omega ; W_{\mathrm{per}, 0}^{1, p}(Y)\right)$ such that along a suitable sequence $\varepsilon_{n} \rightarrow 0$

$$
\nabla u_{\varepsilon_{n}} \stackrel{w-2}{\rightarrow} \nabla u+\nabla_{y} U \quad \text { two-scale weakly in } L^{p}\left(\Omega \times Y ; \mathbb{R}^{N}\right) .
$$

Conversely, for every $u \in W^{1, p}(\Omega)$ and $U \in L^{p}\left(\Omega ; W_{\mathrm{per}, 0}^{1, p}(Y)\right)$, there exists a family $\left(u_{\varepsilon}\right)_{\varepsilon>0}$ in $W^{1, p}(\Omega)$ such that for $\varepsilon \rightarrow 0$

and

$$
u_{\varepsilon} \rightarrow u \quad \text { weakly in } W^{1, p}(\Omega)
$$

$$
\nabla u_{\varepsilon} \stackrel{s-2}{\rightarrow} \nabla u+\nabla_{y} U \quad \text { two-scale strongly in } L^{p}\left(\Omega \times Y ; \mathbb{R}^{N}\right) .
$$

The previous result clearly holds also for the case of sequences.

Remark 3.4. The previous proposition entails the following variant which takes into account boundary conditions. Let $\Omega$ have a Lipschitz boundary. For every $u \in W^{1, p}(\Omega)$ and $U \in L^{p}\left(\Omega ; W_{\text {per, } 0}^{1, p}(Y)\right)$, there exists a family $\left(u_{\varepsilon}\right)_{\varepsilon>0}$ in $W^{1, p}(\Omega)$ with $u_{\varepsilon}=u$ on $\partial \Omega$ (in the sense of traces) for every $\varepsilon>0$ and such that (3.5) and (3.6) hold for $\varepsilon \rightarrow 0$. Indeed, if $\left(\tilde{u}_{\varepsilon}\right)_{\varepsilon>0}$ is the family given by Proposition 3.3, it is sufficient to consider

$$
u_{\varepsilon}:=\varphi_{\varepsilon} \tilde{u}_{\varepsilon}+\left(1-\varphi_{\varepsilon}\right) u,
$$

where $\varphi_{\varepsilon} \in C_{c}^{\infty}(\Omega), 0 \leq \varphi_{\varepsilon} \leq 1$, is such that for $\varepsilon \rightarrow 0$

$$
\varphi_{\varepsilon} \nearrow 1 \text { pointwise in } \Omega \text {, }
$$

and (recall that by compact embedding $\tilde{u}_{\varepsilon} \rightarrow u$ strongly in $L^{p}(\Omega)$ )

$$
\left\|\nabla \varphi_{\varepsilon}\right\|_{\infty}\left\|\tilde{u}_{\varepsilon}-u\right\|_{L^{p}(\Omega)} \rightarrow 0 .
$$


In view of the analysis of the homogenization theory of Fleck and Willis, the following result is essential.

Theorem 3.5. Let $\Omega \subseteq \mathbb{R}^{N}$ be open and bounded with $|\partial \Omega|=0$, and let $\left.p \in\right] 1,+\infty[$. The following facts hold.

(a) If $\left(u_{\varepsilon}\right)_{\varepsilon>0}$ is such that $u_{\varepsilon} \in W^{1, p}(\Omega)$ with

$$
\left\|u_{\varepsilon}\right\|_{L^{p}(\Omega)}+\varepsilon\left\|\nabla u_{\varepsilon}\right\|_{L^{p}\left(\Omega ; \mathbb{R}^{N}\right)} \leq C
$$

for some $C>0$, then there exist $\varepsilon_{n} \rightarrow 0$ and $U \in L^{p}\left(\Omega ; W_{\text {per }}^{1, p}(Y)\right)$ such that

$$
\begin{aligned}
u_{\varepsilon_{n}} \stackrel{w-2}{=} U & \text { two-scale weakly in } L^{p}(\Omega \times Y) \\
\varepsilon_{n} \nabla u_{\varepsilon_{n}} \stackrel{w-2}{=} \nabla_{y} U & \text { two-scale weakly in } L^{p}\left(\Omega \times Y ; \mathbb{R}^{N}\right) .
\end{aligned}
$$

(b) For every $U \in L^{p}\left(\Omega ; W_{\text {per }}^{1, p}(Y)\right)$ there exists a family $\left(u_{\varepsilon}\right)_{\varepsilon>0}$ in $W^{1, p}(\Omega)$ such that for $\varepsilon \rightarrow 0$

$$
\begin{aligned}
u_{\varepsilon} \stackrel{s-2}{\rightarrow} U & \text { two-scale strongly in } L^{p}(\Omega \times Y) \\
\varepsilon \nabla u_{\varepsilon} \stackrel{s-2}{\rightarrow} \nabla_{y} U & \text { two-scale strongly in } L^{p}\left(\Omega \times Y ; \mathbb{R}^{N}\right) .
\end{aligned}
$$

Proof. Point (a) is proved in [1], Proposition 1.14 or [4], Corollary 3.2.

Let us come to point (b). By means of a diagonal argument, it suffices to consider $U$ belonging to the dense subset given by $C_{c}^{1}\left(\Omega ; C_{\mathrm{per}}^{1}(\bar{Y})\right)$. The result follows by setting

$$
u_{\varepsilon}(x):=U\left(x, \frac{x}{\varepsilon}\right) \in C_{c}^{1}(\Omega) .
$$

Indeed, since

$$
\mathcal{T}_{\varepsilon}\left(u_{\varepsilon}\right)(x, y)=U\left(\mathcal{N}_{\varepsilon}(x), y\right),
$$

denoting by $L$ the Lipschitz constant of $U$ we have

$$
\left|\mathcal{T}_{\varepsilon}\left(u_{\varepsilon}\right)(x, y)-U(x, y)\right| \leq L\left|x-\mathcal{N}_{\varepsilon}(x)\right|
$$

so that

We deduce for $\varepsilon \rightarrow 0$

$$
\lim _{\varepsilon \rightarrow 0}\left\|\mathcal{T}_{\varepsilon}\left(u_{\varepsilon}\right)-U\right\|_{L^{p}\left(\mathbb{R}^{N} \times Y\right)}=0 .
$$

$$
u_{\varepsilon} \stackrel{s-2}{\longrightarrow} U \quad \text { two-scale strongly in } L^{p}(\Omega \times Y) .
$$

By the same arguments, since

we infer that for $\varepsilon \rightarrow 0$

$$
\nabla u_{\varepsilon}(x)=\nabla_{x} U\left(x, \frac{x}{\varepsilon}\right)+\frac{1}{\varepsilon} \nabla_{y} U\left(x, \frac{x}{\varepsilon}\right),
$$

so that the proof is concluded.

$$
\varepsilon \nabla u_{\varepsilon} \stackrel{s-2}{\rightarrow} \nabla_{y} U \quad \text { two-scale strongly in } L^{p}\left(\Omega \times Y ; \mathbb{R}^{N}\right),
$$

Remark 3.6 (the vector valued case). The previous results can be adapted to the case of functions taking values in a finite dimensional normed space $X$, since it is sufficient to work component by component.

In particular, we will use the compactness and approximation result of Proposition 3.3 concerning the symmetrized gradient $E u$ of a function $u \in W^{1, p}\left(\Omega ; \mathbb{R}^{N}\right)$. More precisely, a simple symmetrization argument entails the following result. If $\left(u_{\varepsilon}\right)_{\varepsilon>0}$ is a family in $\left.W^{1, p}\left(\Omega ; \mathbb{R}^{N}\right), p \in\right] 1,+\infty\left[\right.$, such that $u_{\varepsilon} \rightarrow u$ weakly in $W^{1, p}\left(\Omega ; \mathbb{R}^{N}\right)$ for $\varepsilon \rightarrow 0$, there exists $U \in L^{p}\left(\Omega ; W_{\text {per }, 0}^{1, p}\left(Y ; \mathbb{R}^{N}\right)\right)$ such that along a suitable sequence $\varepsilon_{n} \rightarrow 0$

$$
E u_{\varepsilon_{n}} \stackrel{w-2}{\longrightarrow} E u+E_{y} U \text { two-scale weakly in } L^{p}\left(\Omega \times Y ; \mathrm{M}_{\mathrm{sym}}^{N}\right) .
$$


Conversely, for every $u \in W^{1, p}\left(\Omega ; \mathbb{R}^{N}\right)$ and $U \in L^{p}\left(\Omega ; W_{\text {per }, 0}^{1, p}\left(Y ; \mathbb{R}^{N}\right)\right)$, there exists a family $\left(u_{\varepsilon}\right)_{\varepsilon>0}$ in $W^{1, p}\left(\Omega ; \mathbb{R}^{N}\right)$ such that for $\varepsilon \rightarrow 0$

$$
u_{\varepsilon} \rightarrow u \quad \text { weakly in } W^{1, p}\left(\Omega ; \mathbb{R}^{N}\right)
$$

and

$$
E u_{\varepsilon} \stackrel{s-2}{\longrightarrow} E u+E_{y} U \quad \text { two-scale strongly in } L^{p}\left(\Omega \times Y ; \mathrm{M}_{\mathrm{sym}}^{N}\right) .
$$

Remark 3.7 (the approximation result under an admissibility constraint). As a consequence of Theorem 3.5 (extended to the vectorial setting according to the previous remark) we get the following approximation result which will be used when dealing with the homogenization of quasistatic evolutions in Section 5 .

Let $\mathrm{M}_{D}^{N}$ and $\mathbb{M}_{D}^{N}$ denote the set of deviatoric matrices defined in Section 2, and let $\ell>0$. If $P \in$ $L^{2}\left(\Omega ; H_{\mathrm{per}}^{1}\left(Y ; \mathrm{M}_{D}^{N}\right)\right)$ and $Z \in L^{2}(\Omega \times Y)$ satisfy

$$
\sqrt{|P|^{2}+\ell^{2}\left|\nabla_{y} P\right|^{2}} \leq Z \quad \text { a.e. in } \Omega \times Y,
$$

for every $\varepsilon>0$ we can find $p_{\varepsilon} \in H^{1}\left(\Omega ; \mathrm{M}_{D}^{N}\right)$ and $z_{\varepsilon} \in L^{2}(\Omega)$ such that

$$
\sqrt{\left|p_{\varepsilon}\right|^{2}+\varepsilon^{2} \ell^{2}\left|\nabla p_{\varepsilon}\right|^{2}} \leq z_{\varepsilon} \quad \text { a.e. in } \Omega,
$$

and as $\varepsilon \rightarrow 0$

$$
\begin{aligned}
p_{\varepsilon} \stackrel{s-2}{\rightarrow} P & \text { two-scale strongly in } L^{2}\left(\Omega \times Y ; \mathrm{M}_{D}^{N}\right) \\
\varepsilon \nabla p_{\varepsilon} \stackrel{s-2}{\rightarrow} \nabla_{y} P & \text { two-scale strongly in } L^{2}\left(\Omega \times Y ; \mathbb{M}_{D}^{N}\right) \\
z_{\varepsilon} \stackrel{s-2}{\rightarrow} Z & \text { two-scale strongly in } L^{2}(\Omega \times Y) .
\end{aligned}
$$

Indeed, let $\left(p_{\varepsilon}\right)_{\varepsilon>0}$ be a family in $H^{1}(\Omega)$ satisfying (3.8) and (3.9) according to Theorem 3.5. Notice that thanks to Jensen's inequality, (3.7) entails

$$
\sqrt{\left|\mathcal{F}_{\varepsilon}(P)\right|^{2}+\ell^{2}\left|\mathcal{F}_{\varepsilon}\left(\nabla_{y} P\right)\right|^{2}} \leq \mathcal{F}_{\varepsilon}(Z) \quad \text { a.e. in } \Omega
$$

where $\mathcal{F}_{\varepsilon}$ is the averaging operator (3.4). We deduce that

$$
\begin{aligned}
\sqrt{\left|p_{\varepsilon}\right|^{2}+\varepsilon^{2} \ell^{2}\left|\nabla p_{\varepsilon}\right|^{2}} & \leq \sqrt{\left|\mathcal{F}_{\varepsilon}(P)\right|^{2}+\ell^{2}\left|\mathcal{F}_{\varepsilon}\left(\nabla_{y} P\right)\right|^{2}}+\left|p_{\varepsilon}-\mathcal{F}_{\varepsilon}(P)\right|+\ell\left|\varepsilon \nabla p_{\varepsilon}-\mathcal{F}_{\varepsilon}\left(\nabla_{y} P\right)\right| \\
& \leq \mathcal{F}_{\varepsilon}(Z)+\left|p_{\varepsilon}-\mathcal{F}_{\varepsilon}(P)\right|+\ell\left|\varepsilon \nabla p_{\varepsilon}-\mathcal{F}_{\varepsilon}\left(\nabla_{y} P\right)\right| .
\end{aligned}
$$

Since by Proposition 3.2

$$
\begin{aligned}
\mathcal{F}_{\varepsilon}(P) \stackrel{s-2}{\rightarrow} P & \text { two-scale strongly in } L^{2}\left(\Omega \times Y ; \mathrm{M}_{D}^{N}\right) \\
\mathcal{F}_{\varepsilon}\left(\nabla_{y} P\right) \stackrel{s-2}{\rightarrow} \nabla_{y} P & \text { two-scale strongly in } L^{2}\left(\Omega \times Y ; \mathbb{M}_{D}^{N}\right) \\
\mathcal{F}_{\varepsilon}\left(z_{\varepsilon}\right) \stackrel{s-2}{\rightarrow} Z & \text { two-scale strongly in } L^{2}(\Omega \times Y),
\end{aligned}
$$

the result follows by choosing

$$
z_{\varepsilon}:=\mathcal{F}_{\varepsilon}(Z)+\left|p_{\varepsilon}-\mathcal{F}_{\varepsilon}(P)\right|+\ell\left|\varepsilon \nabla p_{\varepsilon}-\mathcal{F}_{\varepsilon}\left(\nabla_{y} P\right)\right| .
$$




\section{A TWO-SCALE FRAMEWORK FOR THE HOMOGENIZATION RESUlt of FleCK AND WiLLiS}

The aim of this section is to show that variational arguments based on two-scale convergence provide, in a periodic setting, a rigorous mathematical framework for the homogenization result of Fleck and Willis [9] in strain gradient plasticity.

\subsection{The homogenization result of Fleck and Willis}

Let us briefly describe the result of Fleck and Willis. Let $\Omega \subset \mathbb{R}^{N}$ be the reference configuration of an elastoplastic body subject to infinitesimal displacements. The configuration of $\Omega$ is given by a pair $(u, p)$ where $u: \Omega \rightarrow \mathbb{R}^{N}$ stands for the displacement and $p: \Omega \rightarrow \mathrm{M}_{D}^{N}$ denotes the plastic strain taking values in the space of symmetric deviatoric matrices $\mathrm{M}_{D}^{N}$. The elastic strain of $\Omega$ associated to the configuration $(u, p)$ is then given by

$$
e=E u-p
$$

where $E u$ denotes the symmetrized gradient of $u$.

The elastic properties of $\Omega$ are encoded in the elasticity tensor $\mathbb{C}: \Omega \rightarrow \operatorname{Lin}\left(\mathrm{M}_{\mathrm{sym}}^{N} ; \mathrm{M}_{\mathrm{sym}}^{N}\right)$ which is assumed to satisfy the coercivity condition

$$
\alpha|M|^{2} \leq \mathbb{C}(x) M: M \leq \beta|M|^{2}
$$

for a.e. $x \in \Omega$ and for every $M \in \mathrm{M}_{\text {sym }}^{N}$, where $0<\alpha<\beta<+\infty$.

The plastic behaviour of $\Omega$ is determined by a yielding function $b: \Omega \rightarrow\left[0,+\infty\left[\right.\right.$ such that for a.e. $x \in \mathbb{R}^{N}$

$$
b(x)>c>0 .
$$

The strain gradient deformation theory considered by Fleck and Willis [9] amounts in the minimization of the following energy

$$
\mathcal{E}(u, p):=\frac{1}{2} \int_{\Omega} \mathbb{C}(x)(E u-p):(E u-p) \mathrm{d} x+\int_{\Omega} b(x)\left[|p|^{2}+\ell^{2}|\nabla p|^{2}\right] \mathrm{d} x
$$

under suitable boundary conditions and external loads. Here $\ell>0$ denotes a dissipative length scale which depends on the material under consideration.

Fleck and Willis consider the case of a composite material $\Omega$ whose homogenized response under external loads and prescribed boundary conditions can be described without employing gradients of the plastic strain $p$. The homogenized deformation theory involves the effective energy

$$
\mathcal{E}^{\mathrm{eff}}(u, p)=\int_{\Omega} F^{\mathrm{eff}}(E u(x), p(x)) \mathrm{d} x,
$$

where the effective potential $F^{\mathrm{eff}}(\bar{A}, \bar{p})$ is given by minimizing the energy (4.3) on a representative volume element, among displacement fields $u$ satisfying the linear boundary condition $u=\bar{A} \cdot x$ and plastic strains $p$ whose mean is given precisely by $\bar{p}$.

In the following subsection, we provide a two-scale approach to the homogenization procedure in a periodic setting which justifies in a rigorous mathematical way the effective energy $\mathcal{E}^{\text {eff }}$ (see Thm. 4.3) and provides a cell problem for the energy density $F^{\mathrm{eff}}$ (see Thm. 4.5).

\subsection{Two-scale analysis for the homogenization result of Fleck and Willis}

Let the reference configuration $\Omega \subseteq \mathbb{R}^{N}$ be open, bounded and with Lipschitz boundary. In particular $|\partial \Omega|=0$. by

Let us consider the periodic setting in which the elasticity tensor and the plastic yielding function are provided

$$
\mathbb{C} \in L^{\infty}\left(\mathbb{R}^{N} ; \operatorname{Lin}\left(\mathrm{M}_{\mathrm{sym}}^{N} ; \mathrm{M}_{\mathrm{sym}}^{N}\right)\right) \quad \text { and } \quad b \in L^{\infty}\left(\mathbb{R}^{N}\right)
$$


such that for every $i=1, \ldots, N$ and for a.e. $x \in \mathbb{R}^{N}$

$$
\mathbb{C}\left(x+e_{i}\right)=\mathbb{C}(x) \quad \text { and } \quad b\left(x+e_{i}\right)=b(x),
$$

where $\left\{e_{i}: i=1, \ldots, N\right\}$ denotes the canonical basis of $\mathbb{R}^{N}$. We assume that the coercivity conditions (4.1) and (4.2) hold.

The form of the energy involved in (4.3) suggests the following functional framework for a configuration of $\Omega$ :

$$
u \in H^{1}\left(\Omega ; \mathbb{R}^{N}\right) \quad \text { and } \quad p \in H^{1}\left(\Omega ; \mathrm{M}_{D}^{N}\right) .
$$

The homogenization procedure involves the study of the asymptotic behaviour as $\varepsilon \rightarrow 0$ of an energy of the type

$$
(u, p) \mapsto \frac{1}{2} \int_{\Omega} \mathbb{C}\left(\frac{x}{\varepsilon}\right)(E u-p):(E u-p) \mathrm{d} x+\int_{\Omega} b\left(\frac{x}{\varepsilon}\right)\left[|p|^{2}+\varepsilon^{2} \ell^{2}|\nabla p|^{2}\right] \mathrm{d} x .
$$

Here the elasticity tensor and the yielding function oscillate periodically on a scale $\varepsilon$. Accordingly, the dissipative length scale is given by $\varepsilon \ell$ with $\ell>0$, so that the strain gradient effects tend to vanish in the limit.

Let us consider the functional

$$
\mathcal{E}_{\varepsilon}: H^{1}\left(\Omega ; \mathbb{R}^{N}\right) \times L^{2}\left(\Omega ; \mathrm{M}_{D}^{N}\right) \rightarrow[0,+\infty]
$$

defined as

$$
\mathcal{E}_{\varepsilon}(u, p):=\frac{1}{2} \int_{\Omega} \mathbb{C}\left(\frac{x}{\varepsilon}\right)(E u-p):(E u-p) \mathrm{d} x+\int_{\Omega} b\left(\frac{x}{\varepsilon}\right)\left[|p|^{2}+\varepsilon^{2} \ell^{2}|\nabla p|^{2}\right] \mathrm{d} x
$$

if $p \in H^{1}\left(\Omega ; \mathrm{M}_{D}^{N}\right)$, and $\mathcal{E}_{\varepsilon}(u, p)=+\infty$ if $p \in L^{2}\left(\Omega ; \mathrm{M}_{D}^{N}\right) \backslash H^{1}\left(\Omega ; \mathrm{M}_{D}^{N}\right)$.

In view of the coercivity assumptions on $\mathbb{C}$ and $b$, the inequality

$$
\mathcal{E}_{\varepsilon}\left(u_{\varepsilon}, p_{\varepsilon}\right) \leq C
$$

together with boundary conditions for $u_{\varepsilon}$ entails naturally a bound for $u_{\varepsilon}$ in $H^{1}\left(\Omega ; \mathbb{R}^{N}\right)$ and for $p_{\varepsilon}$ in $L^{2}\left(\Omega ; \mathrm{M}_{D}^{N}\right)$. As a consequence, from a mathematical point of view, the problem of the computation of the effective energy (4.4) can be rephrased as the problem of studying the asymptotic behaviour as $\varepsilon \rightarrow 0$ with respect to the weak topology of $H^{1}\left(\Omega ; \mathbb{R}^{N}\right) \times L^{2}\left(\Omega ; \mathrm{M}_{D}^{N}\right)$ of the minimizers of $\mathcal{E}_{\varepsilon}$, subject to suitable external body forces and boundary conditions.

This goal will be accomplished in Theorem 4.3 by means of a preliminary analysis involving two-scale convergence arguments.

Let us consider the functional

$$
\mathcal{E}: H^{1}\left(\Omega ; \mathbb{R}^{N}\right) \times L^{2}\left(\Omega ; H_{\mathrm{per}, 0}^{1}\left(Y ; \mathbb{R}^{N}\right)\right) \times L^{2}\left(\Omega ; H_{\mathrm{per}}^{1}\left(Y ; \mathrm{M}_{D}^{N}\right)\right) \rightarrow[0,+\infty[
$$

given by

$$
\mathcal{E}(u, U, P):=\frac{1}{2} \int_{\Omega \times Y} \mathbb{C}(y)\left(E u+E_{y} U-P\right):\left(E u+E_{y} U-P\right) \mathrm{d} x \mathrm{~d} y+\int_{\Omega \times Y} b(y)\left[|P|^{2}+\ell^{2}\left|\nabla_{y} P\right|^{2}\right] \mathrm{d} x \mathrm{~d} y,
$$

where $E_{y}$ stands for the symmetrized gradient with respect to $y$.

The following result provides an asymptotic link between $\mathcal{E}_{\varepsilon}$ and $\mathcal{E}$. 
Proposition 4.1. The following facts hold.

(a) Lower estimate: if $\varepsilon_{n} \rightarrow 0$ and $\left(u_{\varepsilon_{n}}, p_{\varepsilon_{n}}\right)_{n \in \mathbb{N}}$ is a sequence in $H^{1}\left(\Omega ; \mathbb{R}^{N}\right) \times H^{1}\left(\Omega ; M_{D}^{N}\right)$ such that

$$
\begin{aligned}
u_{\varepsilon_{n}} \rightarrow u & \text { weakly in } H^{1}\left(\Omega ; \mathbb{R}^{N}\right) \\
E u_{\varepsilon_{n}} \stackrel{w-2}{E} E u+E_{y} U & \text { two-scale weakly in } L^{2}\left(\Omega \times Y ; \mathrm{M}_{\mathrm{sym}}^{N}\right) \\
p_{\varepsilon_{n}} \stackrel{w-2}{=} P & \text { two-scale weakly in } L^{2}\left(\Omega \times Y ; \mathrm{M}_{D}^{N}\right) \\
\varepsilon_{n} \nabla p_{\varepsilon_{n}} \stackrel{w-2}{\longrightarrow} \nabla_{y} P & \text { two-scale weakly in } L^{2}\left(\Omega \times Y ; \mathbb{M}_{D}^{N}\right),
\end{aligned}
$$

then

(b) Recovering family: for every

$$
\mathcal{E}(u, U, P) \leq \liminf _{n \rightarrow \infty} \mathcal{E}_{\varepsilon_{n}}\left(u_{\varepsilon_{n}}, p_{\varepsilon_{n}}\right) .
$$

$$
(u, U, P) \in H^{1}\left(\Omega ; \mathbb{R}^{N}\right) \times L^{2}\left(\Omega ; H_{\mathrm{per}, 0}^{1}\left(Y ; \mathbb{R}^{N}\right)\right) \times L^{2}\left(\Omega ; H_{\mathrm{per}}^{1}\left(Y ; \mathrm{M}_{D}^{N}\right)\right)
$$

there exists a family $\left(u_{\varepsilon}, p_{\varepsilon}\right)_{\varepsilon>0}$ in $H^{1}\left(\Omega ; \mathbb{R}^{N}\right) \times H^{1}\left(\Omega ; \mathrm{M}_{D}^{N}\right)$ such that $u_{\varepsilon}=u$ on $\partial \Omega$ and for $\varepsilon \rightarrow 0$

$$
\begin{aligned}
u_{\varepsilon} \rightarrow u & \text { weakly in } H^{1}\left(\Omega ; \mathbb{R}^{N}\right) \\
E u_{\varepsilon} \stackrel{s-2}{\longrightarrow} E u+E_{y} U & \text { two-scale strongly in } L^{2}\left(\Omega \times Y ; \mathrm{M}_{\mathrm{sym}}^{N}\right) \\
p_{\varepsilon} \stackrel{s-2}{\rightarrow} P & \text { two-scale strongly in } L^{2}\left(\Omega \times Y ; \mathrm{M}_{D}^{N}\right) \\
\varepsilon \nabla p_{\varepsilon} \stackrel{s-2}{\rightarrow} \nabla_{y} P & \text { two-scale strongly in } L^{2}\left(\Omega \times Y ; \mathbb{M}_{D}^{N}\right),
\end{aligned}
$$

so that in particular

$$
\lim _{\varepsilon \rightarrow 0} \mathcal{E}_{\varepsilon}\left(u_{\varepsilon}, p_{\varepsilon}\right)=\mathcal{E}(u, U, P) .
$$

Proof. Point (a) follows immediately observing that

$$
\begin{aligned}
\mathcal{E}_{\varepsilon_{n}}\left(u_{\varepsilon_{n}}, p_{\varepsilon_{n}}\right)= & \frac{1}{2} \int_{\mathbb{R}^{N} \times Y} \mathbb{C}(y)\left(\mathcal{T}_{\varepsilon_{n}}\left(E u_{\varepsilon_{n}}\right)-\mathcal{T}_{\varepsilon_{n}}\left(p_{\varepsilon_{n}}\right)\right):\left(\mathcal{T}_{\varepsilon_{n}}\left(E u_{\varepsilon_{n}}\right)-\mathcal{T}_{\varepsilon_{n}}\left(p_{\varepsilon_{n}}\right)\right) \mathrm{d} x \mathrm{~d} y \\
& +\int_{\mathbb{R}^{N} \times Y} b(y)\left[\left|\mathcal{T}_{\varepsilon_{n}}\left(p_{\varepsilon_{n}}\right)\right|^{2}+\ell^{2}\left|\mathcal{T}_{\varepsilon_{n}}\left(\varepsilon_{n} \nabla p_{\varepsilon_{n}}\right)\right|^{2}\right] \mathrm{d} x \mathrm{~d} y,
\end{aligned}
$$

where $\mathcal{T}_{\varepsilon_{n}}$ is the unfolding operator (3.3), and applying the usual lower semicontinuity for quadratic functionals under weak convergence in $L^{2}\left(\mathbb{R}^{N} \times Y\right)$.

Concerning point (b), by Theorem 3.5 there exists $p_{\varepsilon} \in H^{1}\left(\Omega ; \mathrm{M}_{D}^{N}\right)$ such that for $\varepsilon \rightarrow 0$

$$
p_{\varepsilon} \stackrel{s-2}{\rightarrow} P \quad \text { two-scale strongly in } L^{2}\left(\Omega \times Y ; \mathrm{M}_{D}^{N}\right)
$$

and

$$
\varepsilon \nabla p_{\varepsilon} \stackrel{s-2}{\rightarrow} \nabla_{y} P \quad \text { two-scale strongly in } L^{2}\left(\Omega \times Y ; \mathbb{M}_{D}^{N}\right)
$$

Moreover, by Proposition 3.3 and Remarks 3.4 and 3.6, there exists $u_{\varepsilon} \in H^{1}\left(\Omega ; \mathbb{R}^{N}\right)$ with $u_{\varepsilon}=u$ on $\partial \Omega$ for every $\varepsilon>0$ and such that for $\varepsilon \rightarrow 0$

$$
u_{\varepsilon} \rightarrow u \quad \text { weakly in } H^{1}\left(\Omega ; \mathbb{R}^{N}\right)
$$

and

$$
E u_{\varepsilon} \stackrel{s-2}{\rightarrow} E u+E_{y} U \quad \text { two-scale strongly in } L^{2}\left(\Omega \times Y ; \mathrm{M}_{\mathrm{sym}}^{N}\right) .
$$

The convergence of the energies follows from the representation formula (4.5). 
In order to move toward a single scale setting, let us introduce the functional

$$
\mathcal{E}^{\mathrm{eff}}: H^{1}\left(\Omega ; \mathbb{R}^{N}\right) \times L^{2}\left(\Omega ; \mathrm{M}_{D}^{N}\right) \rightarrow[0,+\infty[
$$

defined by

$$
\begin{aligned}
\mathcal{E}^{\mathrm{eff}}(u, p):=\min _{(U, P)}\left\{\mathcal{E}(u, U, P):(U, P) \in L^{2}\left(\Omega ; H_{\mathrm{per}, 0}^{1}\left(Y ; \mathbb{R}^{N}\right)\right) \times L^{2}\left(\Omega ; H_{\mathrm{per}}^{1}\left(Y ; \mathrm{M}_{D}^{N}\right)\right)\right. \\
\left.\qquad \int_{Y} P(x, y) \mathrm{d} y=p(x) \text { for a.e. } x \in \Omega\right\} .
\end{aligned}
$$

Notice that $U$ is left free in the minimization, while $P(x, y)$ satisfies a constraint on the mean with respect to the microstructural variable $y$.

The minimum in the previous formula is indeed attained as is shown in the following lemma.

Lemma 4.2. Let $(u, p) \in H^{1}\left(\Omega ; \mathbb{R}^{N}\right) \times L^{2}\left(\Omega ; \mathrm{M}_{D}^{N}\right)$. Then there exists a unique pair

$$
(U, P) \in L^{2}\left(\Omega ; H_{\mathrm{per}, 0}^{1}\left(Y ; \mathbb{R}^{N}\right)\right) \times L^{2}\left(\Omega ; H_{\mathrm{per}}^{1}\left(Y ; \mathrm{M}_{D}^{N}\right)\right)
$$

with $\int_{Y} P(x, y) \mathrm{d} y=p(x)$ for a.e. $x \in \Omega$ such that $\mathcal{E}^{\mathrm{eff}}(u, p)=\mathcal{E}(u, U, P)$.

Proof. Let $\left(U_{n}, P_{n}\right)$ be a minimizing sequence for problem (4.6) relative to $(u, p)$. By comparison with the admissible pair given by $(0, p)$, we immediately get that for $n$ large

$$
\frac{1}{2} \int_{\Omega \times Y} \mathbb{C}(y)\left(E u+E_{y} U_{n}-P_{n}\right):\left(E u+E_{y} U_{n}-P_{n}\right) \mathrm{d} x \mathrm{~d} y+\int_{\Omega \times Y} b(y)\left[\left|P_{n}\right|^{2}+\ell^{2}\left|\nabla_{y} P_{n}\right|^{2}\right] \mathrm{d} x \mathrm{~d} y \leq \mathcal{E}(u, 0, p)+1 .
$$

In view of the coercivity assumptions on $\mathbb{C}$ and $b$, up to a subsequence we have that

$$
P_{n} \rightarrow P \quad \text { weakly in } L^{2}\left(\Omega ; H_{\mathrm{per}}^{1}\left(Y ; \mathrm{M}_{D}^{N}\right)\right)
$$

and in view of Korn's inequality for periodic functions with zero mean (see Sect. 2)

$$
U_{n} \rightarrow U \quad \text { weakly in } L^{2}\left(\Omega ; H_{\text {per }, 0}^{1}\left(Y ; \mathbb{R}^{N}\right)\right),
$$

for some $P \in L^{2}\left(\Omega ; H_{\mathrm{per}}^{1}\left(Y ; \mathrm{M}_{D}^{N}\right)\right)$ and $U \in L^{2}\left(\Omega ; H_{\mathrm{per}, 0}^{1}\left(Y ; \mathbb{R}^{N}\right)\right)$. In particular we deduce by lower semicontinuity

$$
\mathcal{E}(u, U, P) \leq \liminf _{n \rightarrow \infty} \mathcal{E}\left(u, U_{n}, P_{n}\right)
$$

Notice that $P(x, y)$ satisfies the constraint concerning the mean with respect to $y$. Indeed, by (4.7) and since $P_{n}$ satisfies the constraint, for every $\varphi \in L^{2}(\Omega)$ we have

$$
\int_{\Omega \times Y} P(x, y) \varphi(x) \mathrm{d} x \mathrm{~d} y=\lim _{n \rightarrow \infty} \int_{\Omega \times Y} P_{n}(x, y) \varphi(x) \mathrm{d} x \mathrm{~d} y=\int_{\Omega} p(x) \varphi(x) \mathrm{d} x,
$$

hence

$$
\int_{Y} P(x, y) \mathrm{d} y=p(x) \quad \text { for a.e. } x \in \Omega \text {. }
$$

The pair $(U, P)$ is thus admissible for $(u, p)$ in (4.6) and

$$
\mathcal{E}^{\mathrm{eff}}(u, p) \leq \mathcal{E}(u, U, P) \leq \liminf _{n \rightarrow \infty} \mathcal{E}\left(u, U_{n}, P_{n}\right)=\mathcal{E}^{\mathrm{eff}}(u, p) .
$$


Then $(U, P)$ is a solution of the minimization problem. Its uniqueness follows by the strict convexity of the functional together with Korn's inequality.

The following theorem shows that $\mathcal{E}^{\text {eff }}$ is indeed the energy associated to the effective behaviour of the material when the strain gradient effects vanish. Let us assume that the boundary displacement is given by the trace on $\partial \Omega$ of a given Sobolev function $\bar{u} \in H^{1}\left(\Omega ; \mathbb{R}^{N}\right)$. Moreover, let us consider body forces acting on $\Omega$ whose density per unit volume is given by a function $f \in L^{2}\left(\Omega ; \mathbb{R}^{N}\right)$.

Theorem 4.3 (the homogenization result of Fleck and Willis). For every $\varepsilon>0$ let $\left(u_{\varepsilon}, p_{\varepsilon}\right)$ be the minimizer of

$$
(u, p) \mapsto \mathcal{E}_{\varepsilon}(u, p)-\int_{\Omega} f \cdot u \mathrm{~d} x
$$

on $H^{1}\left(\Omega ; \mathbb{R}^{N}\right) \times H^{1}\left(\Omega ; \mathrm{M}_{D}^{N}\right)$ with $u=\bar{u}$ on $\partial \Omega$. Then for $\varepsilon \rightarrow 0$

$$
u_{\varepsilon} \rightarrow u_{0} \quad \text { weakly in } H^{1}\left(\Omega ; \mathbb{R}^{N}\right)
$$

and

$$
p_{\varepsilon} \rightarrow p_{0} \quad \text { weakly in } L^{2}\left(\Omega ; \mathrm{M}_{D}^{N}\right),
$$

where $\left(u_{0}, p_{0}\right)$ is the unique minimizer of

$$
(u, p) \mapsto \mathcal{E}^{\mathrm{eff}}(u, p)-\int_{\Omega} f \cdot u \mathrm{~d} x
$$

on $H^{1}\left(\Omega ; \mathbb{R}^{N}\right) \times L^{2}\left(\Omega ; \mathrm{M}_{D}^{N}\right)$ with $u=\bar{u}$ on $\partial \Omega$. Moreover

$$
\lim _{\varepsilon \rightarrow 0} \mathcal{E}_{\varepsilon}\left(u_{\varepsilon}, p_{\varepsilon}\right)=\mathcal{E}^{\mathrm{eff}}\left(u_{0}, p_{0}\right) .
$$

Proof. By comparison with the admissible configuration $(\bar{u}, 0)$ and in view of the coercivity assumptions on $\mathbb{C}$ and $b$ we get

$$
\left\|E u_{\varepsilon}-p_{\varepsilon}\right\|_{L^{2}\left(\Omega ; \mathrm{M}_{\mathrm{sym}}^{N}\right)}^{2}+\left\|p_{\varepsilon}\right\|_{L^{2}\left(\Omega ; \mathrm{M}_{D}^{N}\right)}^{2}+\left\|\varepsilon \nabla p_{\varepsilon}\right\|_{L^{2}\left(\Omega ; \mathbb{M}_{D}^{N}\right)}^{2} \leq \tilde{C}\left(1+\int_{\Omega}|f|\left|u_{\varepsilon}\right| \mathrm{d} x\right)
$$

where $\tilde{C}>0$. In view of Korn's inequality (see Sect. 2 ) we easily obtain

$$
\left\|u_{\varepsilon}\right\|_{H^{1}\left(\Omega ; \mathrm{M}_{\mathrm{sym}}^{N}\right)}^{2}+\left\|p_{\varepsilon}\right\|_{L^{2}\left(\Omega ; \mathrm{M}_{D}^{N}\right)}^{2}+\left\|\varepsilon \nabla p_{\varepsilon}\right\|_{L^{2}\left(\Omega ; \mathbb{M}_{D}^{N}\right)}^{2} \leq C
$$

with $C>0$. We deduce that there exists $\varepsilon_{n} \rightarrow 0$ such that

$$
u_{\varepsilon_{n}} \rightarrow u_{0} \quad \text { weakly in } H^{1}\left(\Omega ; \mathbb{R}^{N}\right)
$$

and thanks to Proposition 3.3 and Remark 3.6

$$
E u_{\varepsilon_{n}} \stackrel{w-2}{\longrightarrow} E u_{0}+E_{y} U_{0} \quad \text { two-scale weakly in } L^{2}\left(\Omega \times Y ; \mathrm{M}_{\mathrm{sym}}^{N}\right)
$$

for some $\left(u_{0}, U_{0}\right) \in H^{1}\left(\Omega ; \mathbb{R}^{N}\right) \times L^{2}\left(\Omega ; H_{\mathrm{per}, 0}^{1}\left(Y ; \mathbb{R}^{N}\right)\right)$. Moreover, in view of Theorem 3.5 , we infer that there exists $P_{0} \in L^{2}\left(\Omega ; H_{\text {per }}^{1}\left(Y ; \mathrm{M}_{D}^{N}\right)\right)$ such that up to a subsequence

$$
p_{\varepsilon_{n}} \stackrel{w-2}{\longrightarrow} P_{0} \quad \text { two-scale weakly in } L^{2}\left(\Omega \times Y ; \mathrm{M}_{D}^{N}\right)
$$


and

$$
\varepsilon_{n} \nabla p_{\varepsilon_{n}} \stackrel{w-2}{\longrightarrow} \nabla_{y} P_{0} \quad \text { two-scale weakly in } L^{2}\left(\Omega \times Y ; \mathbb{M}_{D}^{N}\right) .
$$

The configuration $\left(u_{0}, U_{0}, P_{0}\right)$ is a minimizer of the functional

$$
(u, U, P) \mapsto \mathcal{E}(u, U, P)-\int_{\Omega} f \cdot u \mathrm{~d} x
$$

where $u$ satisfies $u=\bar{u}$ on $\partial \Omega$. Indeed for every admissible $(v, V, Q)$, by Proposition 4.1 there exists a family $\left(v_{\varepsilon}, q_{\varepsilon}\right)_{\varepsilon>0}$ in $H^{1}\left(\Omega ; \mathbb{R}^{N}\right) \times H^{1}\left(\Omega ; \mathrm{M}_{D}^{N}\right)$ with $v_{\varepsilon}=\bar{u}$ on $\partial \Omega$ such that for $\varepsilon \rightarrow 0$

$$
\begin{aligned}
v_{\varepsilon} \rightarrow v & \text { weakly in } H^{1}\left(\Omega ; \mathbb{R}^{N}\right) \\
E v_{\varepsilon} \stackrel{s-2}{\longrightarrow} E v+E_{y} V & \text { two-scale strongly in } L^{2}\left(\Omega \times Y ; \mathrm{M}_{\mathrm{sym}}^{N}\right) \\
q_{\varepsilon} \stackrel{s-2}{\rightarrow} Q & \text { two-scale strongly in } L^{2}\left(\Omega \times Y ; \mathrm{M}_{D}^{N}\right) \\
\varepsilon \nabla q_{\varepsilon} \stackrel{s-2}{\rightarrow} \nabla_{y} Q & \text { two-scale strongly in } L^{2}\left(\Omega \times Y ; \mathbb{M}_{D}^{N}\right),
\end{aligned}
$$

and

Since by minimality of $\left(u_{\varepsilon_{n}}, p_{\varepsilon_{n}}\right)$

$$
\mathcal{E}_{\varepsilon}\left(v_{\varepsilon}, q_{\varepsilon}\right) \rightarrow \mathcal{E}(v, V, Q)
$$

$$
\mathcal{E}_{\varepsilon_{n}}\left(u_{\varepsilon_{n}}, p_{\varepsilon_{n}}\right)-\int_{\Omega} f \cdot u_{\varepsilon_{n}} \mathrm{~d} x \leq \mathcal{E}_{\varepsilon_{n}}\left(v_{\varepsilon_{n}}, q_{\varepsilon_{n}}\right)-\int_{\Omega} f \cdot v_{\varepsilon_{n}} \mathrm{~d} x,
$$

passing to the limit we obtain in view of point (a) of Proposition 4.1

$$
\begin{aligned}
\mathcal{E}\left(u_{0}, U_{0}, P_{0}\right)-\int_{\Omega} f \cdot u_{0} \mathrm{~d} x \leq \liminf _{n \rightarrow \infty} & \left(\mathcal{E}_{\varepsilon_{n}}\left(u_{\varepsilon_{n}}, p_{\varepsilon_{n}}\right)-\int_{\Omega} f \cdot u_{\varepsilon_{n}} \mathrm{~d} x\right) \\
& \leq \limsup _{n \rightarrow \infty}\left(\mathcal{E}_{\varepsilon_{n}}\left(u_{\varepsilon_{n}}, p_{\varepsilon_{n}}\right)-\int_{\Omega} f \cdot u_{\varepsilon_{n}} \mathrm{~d} x\right) \\
& \leq \limsup _{n \rightarrow \infty}\left(\mathcal{E}_{\varepsilon_{n}}\left(v_{\varepsilon_{n}}, q_{\varepsilon_{n}}\right)-\int_{\Omega} f \cdot v_{\varepsilon_{n}} \mathrm{~d} x\right)=\mathcal{E}(v, V, Q)-\int_{\Omega} f \cdot v \mathrm{~d} x .
\end{aligned}
$$

We infer that $\left(u_{0}, U_{0}, P_{0}\right)$ is a minimizer of (4.9). Since the minimizer is unique by strict convexity, we conclude that for $\varepsilon \rightarrow 0$

$$
\begin{aligned}
u_{\varepsilon} \rightarrow u_{0} & \text { weakly in } H^{1}\left(\Omega ; \mathbb{R}^{N}\right) \\
E u_{\varepsilon} \stackrel{w-2}{=} E u_{0}+E_{y} U_{0} & \text { two-scale weakly in } L^{2}\left(\Omega \times Y ; \mathrm{M}_{\mathrm{sym}}^{N}\right) \\
p_{\varepsilon} \stackrel{w-2}{\rightarrow} P_{0} & \text { two-scale weakly in } L^{2}\left(\Omega \times Y ; \mathrm{M}_{D}^{N}\right) \\
\varepsilon \nabla p_{\varepsilon} \stackrel{w-2}{\longrightarrow} \nabla_{y} P_{0} & \text { two-scale weakly in } L^{2}\left(\Omega \times Y ; \mathbb{M}_{D}^{N}\right),
\end{aligned}
$$

and thanks to $(4.10)$ with the choice $(v, V, Q)=\left(u_{0}, U_{0}, P_{0}\right)$

$$
\mathcal{E}_{\varepsilon}\left(u_{\varepsilon}, p_{\varepsilon}\right) \rightarrow \mathcal{E}\left(u_{0}, U_{0}, P_{0}\right)
$$

Let us set for almost every $x \in \Omega$

$$
p_{0}(x):=\int_{Y} P_{0}(x, y) \mathrm{d} y .
$$


Clearly we have $p_{0} \in L^{2}\left(\Omega ; \mathrm{M}_{D}^{N}\right)$. By (3.2) we get

$$
p_{\varepsilon} \rightarrow p_{0} \quad \text { weakly in } L^{2}\left(\Omega ; \mathrm{M}_{D}^{N}\right) .
$$

The result follows provided that we show that the pair $\left(u_{0}, p_{0}\right)$ is the unique minimizer of (4.8) under the boundary condition $u=\bar{u}$ on $\partial \Omega$ with

$$
\mathcal{E}^{\mathrm{eff}}\left(u_{0}, p_{0}\right)=\mathcal{E}\left(u_{0}, U_{0}, P_{0}\right) .
$$

For every $(u, p) \in H^{1}\left(\Omega ; \mathbb{R}^{N}\right) \times L^{2}\left(\Omega ; \mathrm{M}_{D}^{N}\right)$ with $u=\bar{u}$ on $\partial \Omega$, letting $(U, P)$ be the associated pair according to Lemma 4.2 , we have

$$
\mathcal{E}^{\mathrm{eff}}\left(u_{0}, p_{0}\right)-\int_{\Omega} f \cdot u_{0} \mathrm{~d} x \leq \mathcal{E}\left(u_{0}, U_{0}, P_{0}\right)-\int_{\Omega} f \cdot u_{0} \mathrm{~d} x \leq \mathcal{E}(u, U, P)-\int_{\Omega} f \cdot u \mathrm{~d} x=\mathcal{E}^{\mathrm{eff}}(u, p)-\int_{\Omega} f \cdot u \mathrm{~d} x,
$$

so that the minimality of $\left(u_{0}, p_{0}\right)$ follows. The uniqueness holds in view of the strict convexity of $\mathcal{E}$. Indeed, if $(\tilde{u}, \tilde{p})$ were another minimizer, and $(\tilde{U}, \tilde{P})$ the associated pair according to Lemma 4.2 , in view of the preceding inequalities we would get that $(\tilde{u}, \tilde{U}, \tilde{P})$ is a minimizer of (4.9). Since $\mathcal{E}$ is strictly convex, we would infer that $(\tilde{u}, \tilde{U}, \tilde{P})=\left(u_{0}, U_{0}, P_{0}\right)$ so that in particular $\tilde{u}=u_{0}$ and $\tilde{p}=p_{0}$. This entails also that $\left(U_{0}, P_{0}\right)$ is the pair associated to $\left(u_{0}, p_{0}\right)$ according to Lemma 4.2 , so that (4.14) holds, and the proof is concluded.

Remark 4.4. The previous theorem suggests that $\mathcal{E}_{\varepsilon} \Gamma$-converges in the sense of De Giorgi to $\mathcal{E}^{\text {eff }}$ as $\varepsilon \rightarrow 0$. This is indeed the case provided that we consider the weak topology on $H^{1}\left(\Omega ; \mathbb{R}^{N}\right) \times L^{2}\left(\Omega ; \mathrm{M}_{D}^{N}\right)$ and we restrict the functionals to the pairs $(u, p)$ such that $u=\bar{u}$ on $\partial \Omega$. In this way, the convergence of the minimizers $\left(u_{\varepsilon}, p_{\varepsilon}\right)$ along a suitable sequence $\varepsilon_{n} \rightarrow 0$ to a minimizer of the effective energy turns out to be a standard result of $\Gamma$-convergence. The two-scale analysis enables us to deduce that the limit energy has a unique minimizer, so that the convergence holds indeed along the entire family.

In the rest of the section we concentrate on the representation formula (4.4) for $\mathcal{E}^{\mathrm{eff}}$.

Theorem 4.5 (representation formula for the effective energy). For every $(u, p) \in H^{1}\left(\Omega ; \mathbb{R}^{N}\right) \times$ $L^{2}\left(\Omega ; \mathrm{M}_{D}^{N}\right)$ we have

where for $(\bar{A}, \bar{p}) \in \mathrm{M}_{\mathrm{sym}}^{N} \times \mathrm{M}_{D}^{N}$

$$
\mathcal{E}^{\mathrm{eff}}(u, p)=\int_{\Omega} F^{\mathrm{eff}}(E u(x), p(x)) \mathrm{d} x,
$$

$$
\begin{aligned}
F^{\mathrm{eff}}(\bar{A}, \bar{p}):= & \min \left\{\frac{1}{2} \int_{Y} \mathbb{C}(y)\left[\bar{A}+E_{y} U-P\right]:\left[\bar{A}+E_{y} U-P\right] \mathrm{d} y\right. \\
& \left.+\int_{Y} b(y)\left[|P|^{2}+\ell^{2}\left|\nabla_{y} P\right|^{2}\right] \mathrm{d} y:(U, P) \in H_{\mathrm{per}, 0}^{1}\left(Y ; \mathbb{R}^{N}\right) \times H_{\mathrm{per}}^{1}\left(Y ; \mathrm{M}_{D}^{N}\right), \int_{Y} P(y) \mathrm{d} y=\bar{p}\right\} .
\end{aligned}
$$

Proof. Firstly let us prove that the minimum problem in (4.16) admits indeed a unique solution. This follows by the direct method of the Calculus of Variations. If $\left(U_{n}, P_{n}\right)_{n \in \mathbb{N}}$ is a minimizing sequence for the problem, by comparison with the admissible pair $(0, \bar{p})$, taking into account the coercivity assumptions on $\mathbb{C}$ and $b$, one deduces easily that for $n$ large

$$
\left\|E_{y} U_{n}\right\|_{L^{2}\left(Y ; \mathrm{M}_{\mathrm{sym}}^{N}\right)}^{2}+\left\|P_{n}\right\|_{H^{1}\left(Y ; \mathrm{M}_{D}^{N}\right)}^{2} \leq C\left(|\bar{A}|^{2}+|\bar{p}|^{2}\right)
$$

where $C>0$ is a suitable constant. By Korn's inequality (see Sect. 2) we get that $U_{n}$ is bounded in $H^{1}\left(Y ; \mathbb{R}^{N}\right)$. Up to a subsequence we have that

$$
U_{n} \rightarrow U \quad \text { weakly in } H_{\text {per }, 0}^{1}\left(Y ; \mathbb{R}^{N}\right)
$$


and

$$
P_{n} \rightarrow P \quad \text { weakly in } H_{\mathrm{per}}^{1}\left(Y ; \mathrm{M}_{D}^{N}\right) .
$$

Moreover, since $P_{n} \rightarrow P$ strongly in $L^{2}\left(Y ; \mathrm{M}_{D}^{N}\right)$ we get that

$$
\int_{Y} P(y) \mathrm{d} y=\lim _{n \rightarrow \infty} \int_{Y} P_{n}(y) \mathrm{d} y=\bar{p}
$$

We conclude that the pair $(U, P)$ is admissible. By lower semicontinuity, we infer that $(U, P)$ is a minimizer for the functional in (4.16) and satisfies

$$
\left\|E_{y} U\right\|_{L^{2}\left(Y ; \mathrm{M}_{\mathrm{sym}}^{N}\right)}^{2}+\|P\|_{H^{1}\left(Y ; \mathrm{M}_{D}^{N}\right)}^{2} \leq C\left(|\bar{A}|^{2}+|\bar{p}|^{2}\right) .
$$

The uniqueness is ensured by strict convexity.

Let us come to the representation formula (4.15). By Lemma 4.2, for every $(u, p) \in H^{1}\left(\Omega ; \mathbb{R}^{N}\right) \times L^{2}\left(\Omega ; \mathrm{M}_{D}^{N}\right)$ we have

$$
\mathcal{E}^{\mathrm{eff}}(u, p)=\mathcal{E}(u, U, P)
$$

for some $U \in L^{2}\left(\Omega ; H_{\text {per }, 0}^{1}\left(Y ; \mathbb{R}^{N}\right)\right)$ and $P \in L^{2}\left(\Omega ; H_{\text {per }}^{1}\left(Y ; \mathrm{M}_{D}^{N}\right)\right)$ such that

$$
p(x)=\int_{Y} P(x, y) \mathrm{d} y \quad \text { for a.e. } x \in \Omega .
$$

Notice that for a.e. $x \in \Omega$ the pair $(U(x, \cdot), P(x, \cdot))$ is admissible for the computation of $F^{\mathrm{eff}}(E u(x), p(x))$. By the very definition of $F^{\mathrm{eff}}$ we deduce that

$$
\begin{aligned}
\mathcal{E}^{\mathrm{eff}}(u, p) & =\mathcal{E}(u, U, P) \\
=\int_{\Omega}\left[\frac{1}{2} \int_{Y} \mathbb{C}(y)\left[E u(x)+E_{y} U-P\right]:\left[E u(x)+E_{y} U-P\right] \mathrm{d} y+\int_{Y} b(y)\left[|P|^{2}+\ell^{2}\left|\nabla_{y} P\right|^{2}\right] \mathrm{d} y\right] \mathrm{d} x & \geq \int_{\Omega} F^{\mathrm{eff}}(E u(x), p(x)) \mathrm{d} x
\end{aligned}
$$

On the other hand, for a.e. $x \in \Omega$ let $\left(U_{x}, P_{x}\right)$ be the unique solution of problem (4.16) defining $F^{\mathrm{eff}}(E u(x), p(x))$. By (4.17) we deduce that

$$
\left\|E_{y} U_{x}\right\|_{L^{2}\left(Y ; \mathrm{M}_{\mathrm{sym}}^{N}\right)}^{2}+\left\|P_{x}\right\|_{H^{1}\left(Y ; \mathrm{M}_{D}^{N}\right)}^{2} \leq C\left(|E u(x)|^{2}+|p(x)|^{2}\right)
$$

where $C$ does not depend on $x$. We infer that, the measurability with respect to $x$ coming from the uniqueness of the minimizer,

and

$$
U(x, y):=U_{x}(y) \in L^{2}\left(\Omega ; H_{\mathrm{per}, 0}^{1}\left(Y ; \mathbb{R}^{N}\right)\right)
$$

It follows that

$$
P(x, y):=P_{x}(y) \in L^{2}\left(\Omega ; H_{\mathrm{per}}^{1}\left(Y ; \mathrm{M}_{D}^{N}\right)\right) .
$$

and by the very definition of $\mathcal{E}^{\text {eff }}$

$$
\int_{\Omega} F^{\mathrm{eff}}(E u(x), p(x)) \mathrm{d} x=\mathcal{E}(u, U, P),
$$

$$
\int_{\Omega} F^{\mathrm{eff}}(E u(x), p(x)) \mathrm{d} x=\mathcal{E}(u, U, P) \geq \mathcal{E}^{\mathrm{eff}}(u, p) .
$$

In view of (4.18) and (4.19), the representation formula (4.15) follows. 
Let us now investigate the representation formula (4.16) in the particular case when the elastic moduli do not oscillate, i.e.,

$\mathbb{C}$ is constant.

The only term responsible for the homogenization is thus the yielding function $b$. As shown in [9], the representation formula for $F^{\mathrm{eff}}$ involves an operator $\Gamma$ introduced by Willis in [25] which in our context can be characterized as follows.

Definition 4.6. For every $q \in L^{2}\left(Y ; \mathrm{M}_{\mathrm{sym}}^{N}\right)$ let $V$ be the unique minimizer of

$$
V \mapsto \int_{Y} \mathbb{C} E_{y} V:\left[E_{y} V-2 q\right] \mathrm{d} y
$$

on $H_{\mathrm{per}, 0}^{1}\left(Y ; \mathbb{R}^{N}\right)$. We set

$$
\Gamma(\mathbb{C} q):=E_{y} V
$$

so that $\Gamma$ is a well defined operator from $L^{2}\left(Y ; \mathrm{M}_{\mathrm{sym}}^{N}\right)$ into itself.

The effective energy density assumes the following form.

Theorem 4.7. If $\mathbb{C}$ is constant, then for every $(\bar{A}, \bar{p}) \in \mathrm{M}_{\mathrm{sym}}^{N} \times \mathrm{M}_{D}^{N}$ we have

$$
F^{\mathrm{eff}}(\bar{A}, \bar{p})=\frac{1}{2} \mathbb{C}(\bar{A}-\bar{p}):(\bar{A}-\bar{p})+V^{\mathrm{eff}}(\bar{p})
$$

where

$$
\begin{aligned}
V^{\mathrm{eff}}(\bar{p}):=\min \left\{\frac{1}{2} \int_{Y}[\mathbb{C}(P(y)-\bar{p}):(P(y)-\bar{p})-P(y): \mathbb{C} \Gamma(\mathbb{C} P)(y)] \mathrm{d} y\right. & \\
& \quad+\int_{Y} b(y)\left[|P|^{2}+\ell^{2}|\nabla P|^{2} \mathrm{~d} y: P \in H_{\mathrm{per}}^{1}\left(Y ; \mathrm{M}_{D}^{N}\right), \int_{Y} P(y) \mathrm{d} y=\bar{p}\right\},
\end{aligned}
$$

and the operator $\Gamma$ is given in Definition 4.6 .

Proof. If $(U, P)$ is admissible for the computation of $F^{\mathrm{eff}}(\bar{A}, \bar{p})$ according to Theorem 4.5 , since $\mathbb{C}$ is constant, the mean of $P$ on $Y$ is $\bar{p}$, and in view of an integration by parts we have

$$
\begin{aligned}
\frac{1}{2} \int_{Y} \mathbb{C}\left[\bar{A}+E_{y} U-P\right]:\left[\bar{A}+E_{y} U-P\right] \mathrm{d} y= & \frac{1}{2} \int_{Y} \mathbb{C}\left[\bar{A}-\bar{p}+E_{y} U-(P-\bar{p})\right]:\left[\bar{A}-\bar{p}+E_{y} U-(P-\bar{p})\right] \mathrm{d} y \\
= & \frac{1}{2} \mathbb{C}(\bar{A}-\bar{p}):(\bar{A}-\bar{p})+\int_{Y} \mathbb{C}(\bar{A}-\bar{p}): E_{y} U \mathrm{~d} y+\frac{1}{2} \int_{Y} \mathbb{C} E_{y} U: E_{y} U \mathrm{~d} y \\
& -\int_{Y} \mathbb{C} E_{y} U:(P-\bar{p}) \mathrm{d} y+\frac{1}{2} \int_{Y} \mathbb{C}(P-\bar{p}):(P-\bar{p}) \mathrm{d} y \\
= & \frac{1}{2} \mathbb{C}(\bar{A}-\bar{p}):(\bar{A}-\bar{p})+\frac{1}{2} \int_{Y} \mathbb{C}(P-\bar{p}):(P-\bar{p}) \mathrm{d} y \\
& +\frac{1}{2} \int_{Y} \mathbb{C} E_{y} U:\left[E_{y} U-2 P\right] \mathrm{d} y .
\end{aligned}
$$

Taking into account the representation formula (4.16) for $F^{\mathrm{eff}}$ we deduce

$$
\begin{aligned}
F^{\mathrm{eff}}(\bar{A}, \bar{p})=\frac{1}{2} \mathbb{C}(\bar{A}-\bar{p}):(\bar{A}-\bar{p})+\min _{(U, P)}\left[\frac{1}{2} \int_{Y} \mathbb{C}(P-\bar{p}):(P-\bar{p}) \mathrm{d} y+\frac{1}{2} \int_{Y} \mathbb{C} E_{y} U:\left[E_{y} U-2 P\right] \mathrm{d} y\right. \\
\left.+\int_{Y} b(y)\left[|P|^{2}+\ell^{2}|\nabla P|^{2}\right] \mathrm{d} y\right] .
\end{aligned}
$$


We take the minimum on $U$ with $P$ fixed: since $U$ appears only in the second term which attains the minimum for $V$ such that $E_{y} V=\Gamma(\mathbb{C} P)$, with associated value

$$
-\frac{1}{2} \int_{Y} P: \mathbb{C} \Gamma(\mathbb{C} P) \mathrm{d} y
$$

the representation formula follows.

Remark 4.8. When $\mathbb{C}$ is constant the effective energy assumes the form

$$
\mathcal{E}^{\mathrm{eff}}(u, p)=\frac{1}{2} \int_{\Omega} \mathbb{C}(E u(x)-p(x)):(E u(x)-p(x)) \mathrm{d} x+\int_{\Omega} V^{\mathrm{eff}}(p(x)) \mathrm{d} x,
$$

so that it is the sum of an elastic energy and a plastic potential.

The formula suggests that the plastic potential carries an information about the dissipation involved in the plastic process. Notice that $V^{\text {eff }}$ does not only depend on the yielding function $b$, but also on the elasticity tensor $\mathbb{C}$, even if this one is assumed to be constant. This implies that some qualitative properties of the plastic potential, such as growth behaviour at infinity for example, can be different in the homogenized limit.

This fact shows that some problems can occur when dealing with the homogenization of quasistatic evolutions taking the point of view of the energetic approach to rate-independent processes developed by Mielke and his school [16]. Indeed the approach is based on the analysis of deformation-theory type problems where the plastic potential has a linear growth: since the linear growth can be lost in the homogenized limit, the effective plastic potential cannot be interpreted as a dissipation.

We finally note that the interplay between elastic and plastic parts in the definition of $V^{\text {eff }}$ is due to the compatibility condition between elastic and plastic strains, whose sum must be the symmetrized gradient of a displacement (in our treatment such a condition is automatically satisfied since we write $E u-p$ for the elastic strain of $u$ ). Such a condition entails that a decoupling of the problem in elastic and plastic parts cannot be carried out.

\section{Two-SCAle homogenization of A STRAin GRAdient Flow THEORY WITH ISOTROPIC LINEAR HARDENING}

In this section we study the homogenization of a quasistatic evolution for the strain gradient plasticity model studied in Section 4. We consider an evolution with isotropic linear hardening, so that displacements and plastic strains can be described within the mathematical framework of Sobolev spaces introduced before: without hardening, strain localizations may take place, and plastic strains should be described within the theory of functions of bounded variation (see [11]). The model corresponds to a particular case of the one proposed by Gurtin and Anand [13], since we consider only dissipation effects associated to the gradient of the plastic strain. We employ the energetic formulation of rate independent processes due to Mielke and his school (see [16] and references therein).

\subsection{Energetic formulation of a quasistatic evolution}

Let the reference configuration of the elastoplastic body be given by $\Omega \subseteq \mathbb{R}^{N}$ bounded open set with Lipschitz boundary. Let $\partial_{D} \Omega$ be a measurable subset of $\partial \Omega$ with positive surface measure.

A configuration of $\Omega$ is given by a triple $(u, p, z)$ with

$$
u \in H^{1}\left(\Omega ; \mathbb{R}^{N}\right), \quad p \in H^{1}\left(\Omega ; \mathrm{M}_{D}^{N}\right), \quad z \in L^{2}(\Omega),
$$

where $u$ denotes the displacement, $p$ is the associated plastic strain, and $z$ is a hardening internal variable. Here $\mathrm{M}_{D}^{N}$ denotes the space of symmetric deviatoric matrices (see Sect. 2). 
Within the small displacements and small strains approximation, let us consider the free energy

$$
\mathcal{Q}(u, p, z):=\frac{1}{2} \int_{\Omega}\left[\mathbb{C}(x)(E u(x)-p(x)):(E u(x)-p(x))+z^{2}(x)\right] \mathrm{d} x,
$$

where $\mathbb{C} \in L^{\infty}\left(\Omega ; \operatorname{Lin}\left(\mathrm{M}_{\mathrm{sym}}^{N} ; \mathrm{M}_{\mathrm{sym}}^{N}\right)\right)$ denotes an elasticity tensor satisfying the coercivity assumption (4.1).

During the evolution, the higher order stresses associated to $(p, \nabla p)$ (see [13] for their definition) belong to an admissible region $\mathcal{S}_{\text {yield }}$ which becomes larger and larger thanks to the hardening process. We keep track of this fact by considering the convex conjugate of the support function of $\mathcal{S}_{\text {yield }}$ which is given by

$$
\mathcal{H}(p, z):=I_{\mathcal{C}}(p, z)+\int_{\Omega} b(x) z(x) \mathrm{d} x
$$

with $I_{\mathcal{C}}$ denoting the indicator function of the cone

$$
\mathcal{C}:=\left\{(p, z) \in H^{1}\left(\Omega ; \mathrm{M}_{D}^{N}\right) \times L^{2}(\Omega): \sqrt{|p(x)|^{2}+\ell^{2}|\nabla p(x)|^{2}} \leq z(x) \text { for a.e. } x \in \Omega\right\}
$$

where $\ell>0$ is a dissipative length scale, and the yielding function $b \in L^{\infty}(\Omega)$ satisfies the coercivity assumption (4.2).

The dissipation during an evolution $t \mapsto(u(t), p(t), z(t))$ defined on $[0, T]$ relative to a subinterval $[a, b]$ is given in terms of $\mathcal{H}$ by

$$
\mathcal{D}(p, z ; a, b):=\sup \left\{\sum_{j=1}^{k} \mathcal{H}\left(p\left(t_{j}\right)-p\left(t_{j-1}\right), z\left(t_{j}\right)-z\left(t_{j-1}\right)\right): a=t_{0}<\ldots<t_{k}=b\right\} .
$$

An admissible boundary displacement is given by the trace on $\partial_{D} \Omega$ of a function $\psi \in H^{1}\left(\Omega ; \mathbb{R}^{N}\right)$. The family of admissible configurations of $\Omega$ relative to the boundary displacement $\psi$ is then given by

$$
\mathcal{A}(\psi):=\left\{(u, p, z) \in H^{1}\left(\Omega ; \mathbb{R}^{N}\right) \times H^{1}\left(\Omega ; \mathrm{M}_{D}^{N}\right) \times L^{2}(\Omega): u=\psi \text { on } \partial_{D} \Omega,(p, z) \in \mathcal{C}\right\},
$$

where the equality on $\partial_{D} \Omega$ is intended in the sense of traces.

Let us assume that the prescribed boundary displacements on $\partial_{D} \Omega$ are given by the absolutely continuous function

$$
\psi:[0, T] \rightarrow H^{1}\left(\Omega ; \mathbb{R}^{N}\right),
$$

while body and traction forces acting on $\Omega$ are given by the absolutely continuous function

$$
l:[0, T] \rightarrow\left(H^{1}\left(\Omega ; \mathbb{R}^{N}\right)\right)^{*} .
$$

We will denote by $i$ the derivative with respect to $t$ which exists almost everywhere on $[0, T]$.

The energetic formulation of a quasistatic evolution for our model of strain gradient plasticity with isotropic linear hardening is the following.

Definition 5.1 (energetic formulation of a quasistatic evolution). Let $t \mapsto \psi(t)$ and $t \mapsto l(t)$ be assigned boundary displacements and external loads according to (5.2) and (5.3) respectively. A map

$$
\begin{aligned}
{[0, T] } & \rightarrow H^{1}\left(\Omega ; \mathbb{R}^{N}\right) \times H^{1}\left(\Omega ; \mathrm{M}_{D}^{N}\right) \times L^{2}(\Omega) \\
t & \mapsto(u(t), p(t), z(t))
\end{aligned}
$$

is a quasistatic evolution if the following conditions hold for every $t \in[0, T]$. 
(a) Admissibility: $(u(t), p(t), z(t)) \in \mathcal{A}(\psi(t))$.

(b) Global stability: for every $(v, q, \xi) \in \mathcal{A}(\psi(t))$

$$
\mathcal{Q}(u(t), p(t), z(t))-\langle l(t), u(t)\rangle \leq \mathcal{Q}(v, q, \xi)-\langle l(t), v\rangle+\mathcal{H}(q-p(t), \xi-z(t)) .
$$

(c) Energy balance: the function $t \mapsto(p(t), z(t))$ has bounded variation from $[0, T]$ to $H^{1}\left(\Omega ; \mathrm{M}_{D}^{N}\right) \times L^{2}(\Omega)$ and

$$
E(t)+\mathcal{D}(p, z ; 0, t)=E(0)-\int_{0}^{t}\langle\dot{l}(\tau), u(\tau)\rangle \mathrm{d} \tau
$$

where

$$
E(t):=\mathcal{Q}(u(t), p(t), z(t))-\langle l(t), u(t)\rangle,
$$

and $\mathcal{D}(p, z ; 0, t)$ is defined in $(5.1)$.

By general results concerning quasistatic evolutions (see [16] or [17]), the following result holds.

Theorem 5.2. Let $\left(u_{0}, p_{0}, z_{0}\right) \in \mathcal{A}(\psi(0))$ satisfy the global stability condition (5.4). Then there exists a unique quasistatic evolution $t \mapsto(u(t), p(t), z(t))$ such that $(u(0), p(0), z(0))=\left(u_{0}, p_{0}, z_{0}\right)$. Moreover the maps $t \mapsto u(t)$, $t \mapsto p(t)$ and $t \mapsto z(t)$ are absolutely continuous from $[0, T]$ to $H^{1}\left(\Omega ; \mathbb{R}^{N}\right), H^{1}\left(\Omega ; \mathrm{M}_{D}^{N}\right)$ and $L^{2}(\Omega)$ respectively.

Remark 5.3 (connection with the flow rule formulation). The energetic formulation of the evolution is equivalent to the ordinary one involving balance equations for the stresses and the flow rule for the plastic strains. Concerning this issue, the reader is referred to [5] for the case of ordinary plasticity and to [11] for the model of Gurtin and Anand.

Let us briefly summarize the results concerning our framework (for technical details we refer to the above mentioned papers). If the external loads are given by

$$
\langle l(t), u\rangle=\int_{\Omega} f(t) \cdot u \mathrm{~d} x+\int_{\partial_{N} \Omega} g(t) \cdot u \mathrm{~d} S(x)
$$

for suitable body forces $f(t)$ on $\Omega$ and traction forces $g(t)$ on $\partial_{N} \Omega:=\partial \Omega \backslash \partial_{D} \Omega$, the Cauchy stress tensor

$$
\sigma(t):=\mathbb{C}(E u(t)-p(t))
$$

turns out to satisfy for every $t \in[0, T]$ the standard balance equation

$$
\begin{cases}-\operatorname{div} \sigma(t)=f(t) & \text { in } \Omega \\ \sigma(t) \cdot n=g(t) & \text { on } \partial_{N} \Omega .\end{cases}
$$

The higher order stresses $\left(T_{p}(t), \mathbb{K}_{p}(t)\right) \in L^{2}\left(\Omega ; \mathrm{M}_{D}^{N}\right) \times L^{2}\left(\Omega ; \mathbb{M}_{D}^{N}\right)$ associated to $(p(t), \nabla p(t))$ satisfy for every $t \in[0, T]$

$$
\left(T_{p}(t, x), \mathbb{K}_{p}(t, x)\right) \in \mathcal{S}_{\text {yield }}(t, x) \quad \text { for a.e. } x \in \Omega,
$$

where the admissible region $\mathcal{S}_{\text {yield }}(t, x)$ is given by

$$
\mathcal{S}_{\text {yield }}(t, x):=\left\{(A, \mathbb{B}) \in \mathrm{M}_{D}^{N} \times \mathbb{M}_{D}^{N}: \sqrt{|A|^{2}+\frac{1}{\ell^{2}}|\mathbb{B}|^{2}} \leq S_{Y}(t, x)\right\}
$$

with $S_{Y}(t, x):=b(x)+z(t, x)$. Moreover they are related to the Cauchy stress tensor by means of the balance equation

$$
\begin{cases}T_{p}(t)=\sigma_{D}(t)+\operatorname{div} \mathbb{K}_{p}(t) & \text { in } \Omega \\ \mathbb{K}_{p}(t) \cdot n=0 & \text { on } \partial \Omega\end{cases}
$$


where $\sigma_{D}(t)$ denotes the deviatoric part of $\sigma(t)$. Finally, for a.e. $t \in[0, T]$ and for a.e. $x \in \Omega$ the following flow rule holds: if

$$
\sqrt{\left|T_{p}(t, x)\right|^{2}+\frac{1}{\ell^{2}}\left|\mathbb{K}_{p}(t, x)\right|^{2}}<S_{Y}(t, x),
$$

then $(\dot{p}(t, x), \nabla \dot{p}(t, x))=(0,0)$, while if

$$
\sqrt{\left|T_{p}(t, x)\right|^{2}+\frac{1}{\ell^{2}}\left|\mathbb{K}_{p}(t, x)\right|^{2}}=S_{Y}(t, x),
$$

then

$$
\left\{\begin{array}{l}
\dot{p}(t, x)=\lambda(t, x) \frac{T_{p}(t, x)}{\sqrt{\left|T_{p}(t, x)\right|^{2}+\frac{1}{\ell^{2}}\left|\mathbb{K}_{p}(t, x)\right|^{2}}} \\
\nabla \dot{p}(t, x)=\lambda(t, x) \frac{\ell^{-2} \mathbb{K}_{p}(t, x)}{\sqrt{\left|T_{p}(t, x)\right|^{2}+\frac{1}{\ell^{2}}\left|\mathbb{K}_{p}(t, x)\right|^{2}}} \\
\dot{z}(t, x)=\lambda(t, x)
\end{array}\right.
$$

with $\lambda(t, x) \geq 0$.

Notice that for $\ell=0$, the terms involving $\nabla p$ disappear, and the theory formally reduces to the usual von Mises plasticity theory: indeed we have $\mathbb{K}_{p}(t, x)=0$ and $\sigma_{D}(t, x)=T_{p}(t, x)$, with

$$
\left|\sigma_{D}(t, x)\right| \leq S_{Y}(t, x)
$$

Moreover, plasticity develops if $\sigma_{D}(t, x)$ reaches the yield surface, that is if $\left|\sigma_{D}(t, x)\right|=S_{Y}(t, x)$, and in such a case

with $\lambda(t, x) \geq 0$.

$$
\left\{\begin{array}{l}
\dot{p}(t, x)=\lambda(t, x) \frac{\sigma_{D}(t, x)}{\left|\sigma_{D}(t, x)\right|} \\
\dot{z}(t, x)=\lambda(t, x)
\end{array}\right.
$$

\subsection{Homogenization of a quasistatic evolution}

In this subsection we study the asymptotic behaviour of a quasistatic evolution of our strain gradient plasticity model with isotropic linear hardening, in which the elastic and plastic moduli highly oscillate in a periodic way.

Let us assume that the elasticity tensor and the plastic yielding function are provided by

$$
x \mapsto \mathbb{C}\left(\frac{x}{\varepsilon}\right), \quad x \mapsto b\left(\frac{x}{\varepsilon}\right),
$$

where $\varepsilon>0$ and

$$
\mathbb{C} \in L^{\infty}\left(\mathbb{R}^{N} ; \operatorname{Lin}\left(\mathrm{M}_{\mathrm{sym}}^{N} ; \mathrm{M}_{\mathrm{sym}}^{N}\right)\right) \quad \text { and } \quad b \in L^{\infty}\left(\mathbb{R}^{N}\right)
$$

are such that for every $i=1, \ldots, N$ and for a.e. $x \in \mathbb{R}^{N}$

$$
\mathbb{C}\left(x+e_{i}\right)=\mathbb{C}(x) \quad \text { and } \quad b\left(x+e_{i}\right)=b(x) .
$$

Here $\left\{e_{i}: i=1, \ldots, N\right\}$ denotes the canonical basis of $\mathbb{R}^{N}$. We assume that the coercivity conditions (4.1) and (4.2) hold almost everywhere on $\mathbb{R}^{N}$.

We are interested in the asymptotic behaviour of quasistatic evolutions with the choice (5.5), and, as we did in Section 4, with the dissipative length scale of the form $\varepsilon \ell$ with $\ell>0$. Again, it is convenient to move to a two-scale setting. 
A configuration of $\Omega \times Y$, where $Y$ is the unit cell (2.1), is given by

$$
(u, U, P, Z) \in H^{1}\left(\Omega ; \mathbb{R}^{N}\right) \times L^{2}\left(\Omega ; H_{\mathrm{per}, 0}^{1}\left(Y ; \mathbb{R}^{N}\right)\right) \times L^{2}\left(\Omega ; H_{\mathrm{per}}^{1}\left(Y ; \mathbb{R}^{N}\right)\right) \times L^{2}(\Omega \times Y) .
$$

The associated free energy becomes

$$
\tilde{\mathcal{Q}}(u, U, P, Z)=\frac{1}{2} \int_{\Omega \times Y}\left[\mathbb{C}(y)\left(E u+E_{y} U-P\right):\left(E u+E_{y} U-P\right)+Z^{2}(x, y)\right] \mathrm{d} x \mathrm{~d} y,
$$

while the dissipation functional assumes the form

$$
\tilde{\mathcal{H}}(P, Z):=I_{\tilde{\mathcal{C}}}(P, Z)+\int_{\Omega \times Y} b(y) Z \mathrm{~d} x \mathrm{~d} y
$$

with

$\tilde{\mathcal{C}}:=\left\{(P, Z) \in L^{2}\left(\Omega ; H_{\text {per }}^{1}\left(Y ; \mathrm{M}_{D}^{N}\right)\right) \times L^{2}(\Omega \times Y):\right.$

$$
\left.\sqrt{|P(x, y)|^{2}+\ell^{2}\left|\nabla_{y} P(x, y)\right|^{2}} \leq Z(x, y) \text { for a.e. }(x, y) \in \Omega \times Y\right\} .
$$

Let $\tilde{\mathcal{D}}$ be the dissipation associated with $\tilde{\mathcal{H}}$ following the procedure defined in (5.1).

The family of admissible configurations relative to the boundary displacement $\psi$ is given by

$$
\begin{array}{r}
\tilde{\mathcal{A}}(\psi):=\left\{(u, U, P, Z) \in H^{1}\left(\Omega ; \mathbb{R}^{N}\right) \times L^{2}\left(\Omega ; H_{\text {per }, 0}^{1}\left(Y ; \mathbb{R}^{N}\right)\right) \times L^{2}\left(\Omega ; H_{\text {per }}^{1}\left(Y ; \mathbb{R}^{N}\right)\right) \times L^{2}(\Omega \times Y):\right. \\
\left.u=\psi \text { on } \partial_{D} \Omega \text { and }(P, Z) \in \tilde{\mathcal{C}}\right\} .
\end{array}
$$

Definition 5.4 (two-scale quasistatic evolution). Let $t \mapsto \psi(t)$ and $t \mapsto l(t)$ be assigned boundary displacements and external loads according to (5.2) and (5.3) respectively. A map

$$
\begin{aligned}
{[0, T] } & \rightarrow H^{1}\left(\Omega ; \mathbb{R}^{N}\right) \times L^{2}\left(\Omega ; H_{\mathrm{per}, 0}^{1}\left(Y ; \mathbb{R}^{N}\right)\right) \times L^{2}\left(\Omega ; H_{\mathrm{per}}^{1}\left(Y ; \mathrm{M}_{D}^{N}\right)\right) \times L^{2}(\Omega \times Y) \\
t & \mapsto(u(t), U(t), P(t), Z(t))
\end{aligned}
$$

is a quasistatic evolution if the following conditions hold for every $t \in[0, T]$.

(a) Admissibility: $(u(t), U(t), P(t), Z(t)) \in \tilde{\mathcal{A}}(\psi(t))$.

(b) Global stability: for every $(v, V, Q, \Xi) \in \tilde{\mathcal{A}}(\psi(t))$

$$
\tilde{\mathcal{Q}}(u(t), U(t), P(t), Z(t))-\langle l(t), u(t)\rangle \leq \tilde{\mathcal{Q}}(v, V, Q, \Xi)-\langle l(t), v\rangle+\tilde{\mathcal{H}}(Q-P(t), \Xi-Z(t)) .
$$

(c) Energy balance: the function $t \mapsto(P(t), Z(t))$ has bounded variation from $[0, T]$ to $L^{2}\left(\Omega ; H_{\text {per }}^{1}\left(Y ; \mathrm{M}_{D}^{N}\right)\right) \times L^{2}(\Omega \times Y)$ and

$$
\tilde{E}(t)+\tilde{\mathcal{D}}(P, Z ; 0, t)=\tilde{E}(0)-\int_{0}^{t}\langle\dot{l}(\tau), u(\tau)\rangle \mathrm{d} \tau
$$

where

$$
\tilde{E}(t):=\tilde{\mathcal{Q}}(u(t), U(t), P(t), Z(t))-\langle l(t), u(t)\rangle .
$$


The following existence result holds (see [16] or [17]).

Theorem 5.5. Let $\left(u_{0}, U_{0}, P_{0}, Z_{0}\right) \in \tilde{\mathcal{A}}(\psi(0))$ satisfy the global stability condition (5.6). Then there exists a unique quasistatic evolution $t \mapsto(u(t), U(t), P(t), Z(t))$ such that

$$
(u(0), U(0), P(0), Z(0))=\left(u_{0}, U_{0}, P_{0}, Z_{0}\right) .
$$

Moreover the maps $t \mapsto u(t), t \mapsto U(t), t \mapsto P(t)$ and $t \mapsto Z(t)$ are absolutely continuous from [0,T] to $H^{1}\left(\Omega ; \mathbb{R}^{N}\right), L^{2}\left(\Omega ; H_{\text {per }, 0}^{1}\left(Y ; \mathrm{M}_{D}^{N}\right)\right), L^{2}\left(\Omega ; H_{\text {per }}^{1}\left(Y ; \mathrm{M}_{D}^{N}\right)\right)$ and $L^{2}(\Omega \times Y)$ respectively.

Coming back to our model with the choices (5.5) for the elasticity tensor and the yielding function, with dissipative length scale $\varepsilon \ell$, let us denote by $\mathcal{Q}_{\varepsilon}, \mathcal{H}_{\varepsilon}, \mathcal{D}_{\varepsilon}, E_{\varepsilon}$ the associated free energy, dissipation functionals and total energy respectively. Moreover $\mathcal{C}_{\varepsilon}$ will denote the cone associated to $\mathcal{H}_{\varepsilon}$, and $\mathcal{A}_{\varepsilon}(\psi)$ the family of admissible configurations relative to $\psi$.

For every $\varepsilon>0$ let

$$
\left(u_{\varepsilon}^{0}, p_{\varepsilon}^{0}, z_{\varepsilon}^{0}\right) \in \mathcal{A}_{\varepsilon}(\psi(0))
$$

be globally stable initial configurations such that

$$
\begin{cases}u_{\varepsilon}^{0} \rightarrow u_{0} & \text { weakly in } H^{1}\left(\Omega ; \mathbb{R}^{N}\right) \\ E u_{\varepsilon}^{0} \stackrel{w-2}{\longrightarrow} E u_{0}+E_{y} U_{0} & \text { two-scale weakly in } L^{2}\left(\Omega \times Y ; \mathrm{M}_{\mathrm{sym}}^{N}\right) \\ p_{\varepsilon}^{0} \stackrel{w-2}{\longrightarrow} P_{0} & \text { two-scale weakly in } L^{2}\left(\Omega \times Y ; \mathrm{M}_{D}^{N}\right) \\ \varepsilon \nabla p_{\varepsilon}^{0} \stackrel{w-2}{\longrightarrow} \nabla_{y} P_{0} & \text { two-scale weakly in } L^{2}\left(\Omega \times Y ; \mathbb{M}_{D}^{N}\right) \\ z_{0}^{\varepsilon} \stackrel{w-2}{\longrightarrow} Z_{0} & \text { two-scale weakly in } L^{2}(\Omega \times Y)\end{cases}
$$

for some

$$
\left(u_{0}, U_{0}, P_{0}, Z_{0}\right) \in H^{1}\left(\Omega ; \mathbb{R}^{N}\right) \times L^{2}\left(\Omega ; H_{\mathrm{per}, 0}^{1}\left(Y ; \mathbb{R}^{N}\right)\right) \times L^{2}\left(\Omega ; H_{\mathrm{per}}^{1}\left(Y ; \mathbb{R}^{N}\right)\right) \times L^{2}(\Omega \times Y) .
$$

Lemma 5.6. The configuration $\left(u_{0}, U_{0}, P_{0}, Z_{0}\right)$ is admissible for $\psi(0)$ and globally stable according to (5.6).

Proof. The condition $u_{0}=\psi(0)$ on $\partial_{D} \Omega$ comes from the strong convergence for the traces of $u_{\varepsilon}^{0}$. The admissibility thus follows if we prove that $\left(P_{0}, Z_{0}\right) \in \tilde{\mathcal{C}}$. This comes from the inclusion

$$
\left(\mathcal{T}_{\varepsilon}\left(p_{\varepsilon}^{0}\right), \mathcal{T}_{\varepsilon}\left(z_{\varepsilon}^{0}\right)\right) \in \tilde{\mathcal{C}}
$$

$\mathcal{T}_{\varepsilon}$ being the unfolding operator (3.3), together with the fact that the convex cone $\tilde{\mathcal{C}}$ is weakly closed.

Let us prove the global stability condition. Given $(v, V, Q, \Xi) \in \tilde{\mathcal{A}}(\psi(0))$, we want to show that

$$
\tilde{\mathcal{Q}}\left(u_{0}, U_{0}, P_{0}, Z_{0}\right)-\left\langle l(0), u_{0}\right\rangle \leq \tilde{\mathcal{Q}}(v, V, Q, \Xi)-\langle l(0), v\rangle+\tilde{\mathcal{H}}\left(Q-P_{0}, \Xi-Z_{0}\right)
$$

We may assume that $\tilde{\mathcal{H}}\left(Q-P_{0}, \Xi-Z_{0}\right)<+\infty$, so that

$$
\sqrt{\left|Q-P_{0}\right|^{2}+\ell^{2}\left|\nabla_{y} Q-\nabla_{y} P_{0}\right|^{2}} \leq \Xi-Z_{0} \quad \text { a.e. in } \Omega \times Y .
$$

In view of Remark 3.7, we can find $q_{\varepsilon} \in H^{1}\left(\Omega ; \mathrm{M}_{D}^{N}\right)$ and $\xi_{\varepsilon} \in L^{2}(\Omega)$ such that for $\varepsilon \rightarrow 0$

$$
\begin{gathered}
q_{\varepsilon} \stackrel{s-2}{\rightarrow} Q-P_{0} \quad \text { two-scale strongly in } L^{2}\left(\Omega \times Y ; \mathrm{M}_{D}^{N}\right) \\
\varepsilon \nabla q_{\varepsilon} \stackrel{s-2}{\rightarrow} \nabla_{y} Q-\nabla_{y} P_{0} \quad \text { two-scale strongly in } L^{2}\left(\Omega \times Y ; \mathbb{M}_{D}^{N}\right) \\
\xi_{\varepsilon} \stackrel{s-2}{\longrightarrow} \Xi-Z_{0} \quad \text { two-scale strongly in } L^{2}(\Omega \times Y)
\end{gathered}
$$


and

$$
\sqrt{\left|q_{\varepsilon}\right|^{2}+\varepsilon^{2} \ell^{2}\left|\nabla q_{\varepsilon}\right|^{2}} \leq \xi_{\varepsilon} \quad \text { a.e. in } \Omega .
$$

This implies (using Prop. 3.2, point (4)) that

$$
\lim _{\varepsilon \rightarrow 0} \mathcal{H}_{\varepsilon}\left(q_{\varepsilon}, \xi_{\varepsilon}\right)=\tilde{\mathcal{H}}\left(Q-P_{0}, \Xi-Z_{0}\right)
$$

By Proposition 3.3 and Remarks 3.4 and 3.6, we can find $v_{\varepsilon} \in H^{1}\left(\Omega ; \mathbb{R}^{N}\right)$ such that for $\varepsilon \rightarrow 0$

$$
v_{\varepsilon} \rightarrow v-u_{0} \quad \text { weakly in } H^{1}\left(\Omega ; \mathbb{R}^{N}\right),
$$

$v_{\varepsilon}=v-u_{0}$ on $\partial \Omega$ and

$$
E v_{\varepsilon} \stackrel{s-2}{\rightarrow} E v-E u_{0}+E_{y} V-E_{y} U_{0} \quad \text { two-scale strongly in } L^{2}\left(\Omega \times Y ; \mathrm{M}_{\mathrm{sym}}^{N}\right)
$$

By comparing $\left(u_{\varepsilon}^{0}, p_{\varepsilon}^{0}, z_{\varepsilon}^{0}\right)$ with $\left(v_{\varepsilon}+u_{\varepsilon}^{0}, q_{\varepsilon}+p_{\varepsilon}^{0}, \xi_{\varepsilon}+z_{\varepsilon}^{0}\right) \in \mathcal{A}_{\varepsilon}(\psi(0))$ we get

$$
\mathcal{Q}_{\varepsilon}\left(u_{\varepsilon}^{0}, p_{\varepsilon}^{0}, z_{\varepsilon}^{0}\right)-\left\langle l(0), u_{\varepsilon}^{0}\right\rangle \leq \mathcal{Q}_{\varepsilon}\left(v_{\varepsilon}+u_{\varepsilon}^{0}, q_{\varepsilon}+p_{\varepsilon}^{0}, \xi_{\varepsilon}+z_{\varepsilon}^{0}\right)-\left\langle l(0), v_{\varepsilon}+u_{\varepsilon}^{0}\right\rangle+\mathcal{H}_{\varepsilon}\left(q_{\varepsilon}, \xi_{\varepsilon}\right)
$$

Expanding the terms of the free energy and erasing the quadratic terms involving $p_{\varepsilon}^{0}$ and $z_{\varepsilon}^{0}$ we obtain

$$
\begin{array}{r}
0 \leq \frac{1}{2} \int_{\Omega} \mathbb{C}\left(\frac{x}{\varepsilon}\right)\left[E v_{\varepsilon}-q_{\varepsilon}\right]:\left[E v_{\varepsilon}-q_{\varepsilon}\right] \mathrm{d} x+\int_{\Omega} \mathbb{C}\left(\frac{x}{\varepsilon}\right)\left[E v_{\varepsilon}-q_{\varepsilon}\right]:\left[E u_{\varepsilon}^{0}-p_{\varepsilon}^{0}\right] \mathrm{d} x \\
\quad+\frac{1}{2} \int_{\Omega} \xi_{\varepsilon}^{2} \mathrm{~d} x+\int_{\Omega} \xi_{\varepsilon} z_{\varepsilon}^{0} \mathrm{~d} x-\left\langle l(0), v_{\varepsilon}\right\rangle+\mathcal{H}_{\varepsilon}\left(q_{\varepsilon}, \xi_{\varepsilon}\right) .
\end{array}
$$

Letting $\varepsilon \rightarrow 0$ we obtain (using Prop. 3.2, points (3) and (4))

$$
\begin{aligned}
0 \leq \frac{1}{2} \int_{\Omega \times Y} \mathbb{C}(y)\left[E v-E u_{0}+\right. & \left.E_{y} V-E_{y} U_{0}-Q+P_{0}\right]:\left[E v-E u_{0}+E_{y} V-E_{y} U_{0}-Q+P_{0}\right] \mathrm{d} x \mathrm{~d} y \\
+\int_{\Omega \times Y} \mathbb{C}(y)\left[E v-E u_{0}+E_{y} V-E_{y} U_{0}-Q+P_{0}\right]:\left[E u_{0}+E_{y} U_{0}-P_{0}\right] \mathrm{d} x \mathrm{~d} y & \\
& +\frac{1}{2} \int_{\Omega \times Y}\left(\Xi-Z_{0}\right)^{2} \mathrm{~d} x \mathrm{~d} y+\int_{\Omega \times Y}\left(\Xi-Z_{0}\right) Z_{0} \mathrm{~d} x \mathrm{~d} y \\
& -\left\langle l(0), v-u_{0}\right\rangle+\tilde{\mathcal{H}}\left(Q-P_{0}, \Xi-Z_{0}\right),
\end{aligned}
$$

so that, adding to both sides $\tilde{\mathcal{Q}}\left(u_{0}, U_{0}, P_{0}, Z_{0}\right)$ we get precisely the global stability (5.8).

We assume moreover that

$$
\lim _{\varepsilon \rightarrow 0}\left(\mathcal{Q}_{\varepsilon}\left(u_{\varepsilon}^{0}, p_{\varepsilon}^{0}, z_{\varepsilon}^{0}\right)-\left\langle l(0), u_{\varepsilon}^{0}\right\rangle\right)=\tilde{\mathcal{Q}}\left(u_{0}, U_{0}, P_{0}, Z_{0}\right)-\left\langle l(0), u_{0}\right\rangle
$$

Remark 5.7. Notice that the case of purely elastic initial configurations fulfill the global stability condition and our assumptions (5.7) and (5.9).

More precisely, let $u_{\varepsilon}^{0} \in H^{1}\left(\Omega ; \mathbb{R}^{N}\right)$ be the elastic configuration associated to the boundary displacement $\psi(0)$ and the external load $l(0)$, such that the associated Cauchy stress $\sigma_{\varepsilon}^{0}$ satisfies

$$
\left|\left(\sigma_{\varepsilon}^{0}\right)_{D}\right| \leq b\left(\frac{x}{\varepsilon}\right) \quad \text { for a.e. } x \in \Omega
$$


for every $\varepsilon>0$. Then the initial configuration

$$
\left(u_{\varepsilon}^{0}, 0,0\right)
$$

satisfies (5.7) and (5.9) with respect to $\left(u_{0}, U_{0}, 0,0\right)$, for suitable

$$
u_{0} \in H^{1}\left(\Omega ; \mathbb{R}^{N}\right) \quad \text { and } \quad U_{0} \in L^{2}\left(\Omega ; H_{\mathrm{per}, 0}^{1}\left(Y ; \mathbb{R}^{N}\right)\right) .
$$

The global stability condition follows since $\left(u_{\varepsilon}^{0}, 0,0\right)$ is the minimizer of the convex function

$$
(u, p, z) \mapsto \mathcal{Q}_{\varepsilon}(u, p, z)-\langle l(0), u\rangle+\mathcal{H}_{\varepsilon}(p, z)
$$

The minimality is consequence of (5.10) which entails

$$
-\partial Q_{\varepsilon}\left(u_{\varepsilon}^{0}, 0,0\right)+l(0) \in \mathcal{C}_{\varepsilon}
$$

Thanks to Lemma 5.6, $\left(u_{0}, U_{0}, P_{0}, Z_{0}\right) \in \tilde{\mathcal{A}}(\psi(0))$ is a globally stable configuration, so that the associated two-scale quasistatic evolution is well defined. We are now in a position to state the main result of the section.

Theorem 5.8 (asymptotic behaviour of a quasistatic evolution). Let

$$
t \mapsto\left(u_{\varepsilon}(t), p_{\varepsilon}(t), z_{\varepsilon}(t)\right)
$$

be the quasistatic evolution with initial configuration $\left(u_{\varepsilon}^{0}, p_{\varepsilon}^{0}, z_{\varepsilon}^{0}\right)$ satisfying (5.7) and (5.9). Let

$$
t \mapsto(u(t), U(t), P(t), Z(t))
$$

be the two-scale quasistatic evolution with initial configuration $\left(u_{0}, U_{0}, P_{0}, Z_{0}\right)$.

Then for every $t \in[0, T]$

$$
\begin{aligned}
u_{\varepsilon}(t) \rightarrow u(t) & \text { weakly in } H^{1}\left(\Omega ; \mathbb{R}^{N}\right) \\
E u_{\varepsilon}(t) \stackrel{w-2}{\longrightarrow} E u(t)+E_{y} U(t) & \text { two-scale weakly in } L^{2}\left(\Omega \times Y ; \mathrm{M}_{\mathrm{sym}}^{N}\right) \\
p_{\varepsilon}(t) \stackrel{w-2}{\longrightarrow} P(t) & \text { two-scale weakly in } L^{2}\left(\Omega \times Y ; \mathrm{M}_{D}^{N}\right) \\
\varepsilon \nabla p_{\varepsilon}(t) \stackrel{w-2}{\longrightarrow} \nabla_{y} P(t) & \text { two-scale weakly in } L^{2}\left(\Omega \times Y ; \mathbb{M}_{D}^{N}\right) \\
z_{\varepsilon}(t) \stackrel{s-2}{\rightarrow} Z(t) & \text { two-scale strongly in } L^{2}(\Omega \times Y) .
\end{aligned}
$$

Finally, concerning the elastic strain we have for every $t \in[0, T]$

$$
E u_{\varepsilon}(t)-p_{\varepsilon}(t) \stackrel{s-2}{\rightarrow} E u(t)+E_{y} U(t)-P(t) \quad \text { two-scale strongly in } L^{2}\left(\Omega \times Y ; \mathrm{M}_{\mathrm{sym}}^{N}\right) \text {. }
$$

Proof. We divide the proof in several steps.

Step 1: Compactness for the plastic strain and the hardening variable. From the energy balance

$$
E_{\varepsilon}(t)+\mathcal{D}_{\varepsilon}\left(p_{\varepsilon}, z_{\varepsilon} ; 0, t\right)=E_{\varepsilon}(0)-\int_{0}^{t}\left\langle\dot{l}(\tau), u_{\varepsilon}(\tau)\right\rangle \mathrm{d} \tau
$$

and recalling that by $(5.9)$

$$
E_{\varepsilon}(0)=\mathcal{Q}_{\varepsilon}\left(u_{\varepsilon}^{0}, p_{\varepsilon}^{0}, z_{\varepsilon}^{0}\right)-\left\langle l(0), u_{\varepsilon}^{0}\right\rangle \rightarrow \tilde{\mathcal{Q}}\left(u_{0}, U_{0}, P_{0}, Z_{0}\right)-\left\langle l(0), u_{0}\right\rangle \quad \text { as } \varepsilon \rightarrow 0,
$$


in view of the coercivity assumptions for the elastic and plastic moduli we obtain for every $t \in[0, T]$ and for $\varepsilon>0$ small enough

$$
\left\|E u_{\varepsilon}(t)-p_{\varepsilon}(t)\right\|_{L^{2}\left(\Omega ; \mathrm{M}_{\mathrm{sym}}^{N}\right)}^{2}+\left\|z_{\varepsilon}(t)\right\|_{L^{2}(\Omega)}^{2} \leq C\left(1+\max _{\tau \in[0, t]}\left\|u_{\varepsilon}(\tau)\right\|_{H^{1}\left(\Omega ; \mathbb{R}^{N}\right)}\right)
$$

where $C>0$ is a suitable constant independent of $\varepsilon$ and $t$. Since $\left(u_{\varepsilon}(t), p_{\varepsilon}(t), z_{\varepsilon}(t)\right) \in \mathcal{A}_{\varepsilon}(\psi(t))$ we get up to changing $C$

$$
\left\|E u_{\varepsilon}(t)\right\|_{L^{2}\left(\Omega ; \mathrm{M}_{\mathrm{sym}}^{N}\right)}^{2}+\left\|z_{\varepsilon}(t)\right\|_{L^{2}(\Omega)}^{2} \leq C\left(1+\max _{\tau \in[0, t]}\left\|u_{\varepsilon}(\tau)\right\|_{H^{1}\left(\Omega ; \mathbb{R}^{N}\right)}\right)
$$

so that we infer in view of Korn's inequality (see Sect. 2)

$$
\max _{t \in[0, T]}\left\|E u_{\varepsilon}(t)\right\|_{L^{2}\left(\Omega ; \mathrm{M}_{\mathrm{sym}}^{N}\right)}^{2} \leq C\left(1+\max _{t \in[0, T]}\left\|E u_{\varepsilon}(t)\right\|_{L^{2}\left(\Omega ; \mathrm{M}_{\mathrm{sym}}^{N}\right)}\right) .
$$

This entails, by (5.11) and using again Korn's inequality, that for $\varepsilon$ small enough the quantity

$$
E_{\varepsilon}(t)+\mathcal{D}_{\varepsilon}\left(p_{\varepsilon}, z_{\varepsilon} ; 0, t\right)
$$

is uniformly bounded for $t \in[0, T]$.

Taking into account the definition of $\mathcal{D}_{\varepsilon}\left(p_{\varepsilon}, z_{\varepsilon} ; 0, t\right)$, and using the coercivity for the yielding function $b$, we infer that the total variation of

$$
t \mapsto z_{\varepsilon}(t)
$$

from $[0, T]$ to $L^{2}(\Omega)$ is uniformly bounded for $\varepsilon$ small. By admissibility of the configurations, we deduce also that the total variation of

$$
t \mapsto\left(p_{\varepsilon}(t), \varepsilon \nabla p_{\varepsilon}(t)\right)
$$

from $[0, T]$ to $L^{2}\left(\Omega ; \mathrm{M}_{D}^{N}\right) \times L^{2}\left(\Omega ; \mathbb{M}_{D}^{N}\right)$ is uniformly bounded for $\varepsilon$ small.

From the bound on $E_{\varepsilon}(t)$, using again Korn's inequality and the admissibility of the configurations, we infer that there exists $\tilde{C}>0$ such that for $\varepsilon$ small enough and for every $t \in[0, T]$

$$
\left\|u_{\varepsilon}(t)\right\|_{H^{1}\left(\Omega ; \mathbb{R}^{N}\right)}+\left\|p_{\varepsilon}(t)\right\|_{L^{2}\left(\Omega ; \mathrm{M}_{D}^{N}\right)}+\left\|\varepsilon \nabla p_{\varepsilon}(t)\right\|_{L^{2}\left(\Omega ; \mathbb{M}_{D}^{N}\right)}+\left\|z_{\varepsilon}(t)\right\|_{L^{2}(\Omega)} \leq \tilde{C} .
$$

Since the unfolding operator $\mathcal{T}_{\varepsilon}$ is an isometry, we deduce that the total variation of

$$
t \mapsto\left(\mathcal{T}_{\varepsilon}\left(p_{\varepsilon}(t)\right), \mathcal{T}_{\varepsilon}\left(\varepsilon \nabla p_{\varepsilon}(t)\right), \mathcal{T}_{\varepsilon}\left(z_{\varepsilon}(t)\right)\right)
$$

on $[0, T]$ with values in $L^{2}\left(\Omega \times Y ; \mathrm{M}_{D}^{N}\right) \times L^{2}\left(\Omega \times Y ; \mathbb{M}_{D}^{N}\right) \times L^{2}(\Omega \times Y)$ is uniformly bounded for $\varepsilon$ small. By the generalized version of Helly's theorem [5], Lemma 7.2, and in view of Theorem 3.5, we deduce that there exist a function of bounded variation

$$
t \mapsto(P(t), Z(t)) \in H_{\mathrm{per}}^{1}\left(Y ; \mathrm{M}_{D}^{N}\right) \times L^{2}(\Omega \times Y)
$$

and a sequence $\varepsilon_{n} \rightarrow 0$ such that setting

$$
\left(u_{n}(t), p_{n}(t), z_{n}(t)\right):=\left(u_{\varepsilon_{n}}(t), p_{\varepsilon_{n}}(t), z_{\varepsilon_{n}}(t)\right),
$$

for every $t \in[0, T]$

and

$$
\begin{gathered}
p_{n}(t) \stackrel{\text { w-2 }}{\longrightarrow} P(t) \quad \text { two-scale weakly in } L^{2}\left(\Omega \times Y ; \mathrm{M}_{D}^{N}\right), \\
\varepsilon_{n} \nabla p_{n}(t) \stackrel{w-2}{\longrightarrow} \nabla_{y} P(t) \quad \text { two-scale weakly in } L^{2}\left(\Omega \times Y ; \mathbb{M}_{D}^{N}\right),
\end{gathered}
$$

$$
z_{n}(t) \stackrel{w-2}{=} Z(t) \quad \text { two-scale weakly in } L^{2}(\Omega \times Y) .
$$

Notice that from the admissibility of $\left(u_{\varepsilon}(t), p_{\varepsilon}(t), z_{\varepsilon}(t)\right)$ we infer that $(P(t), Z(t)) \in \tilde{\mathcal{C}}$ for every $t \in[0, T]$. 
Step 2: Compactness for the displacement. Let us fix $t \in[0, T]$. In view of (5.12) and Proposition 3.3, up to a further subsequence we have that

for some

$$
\begin{gathered}
u_{n}(t) \rightarrow \tilde{u} \quad \text { weakly in } H^{1}\left(\Omega ; \mathbb{R}^{N}\right) \\
E u_{n}(t) \stackrel{w-2}{\longrightarrow} E \tilde{u}+E_{y} \tilde{U} \quad \text { two-scale weakly in } L^{2}\left(\Omega \times Y ; \mathrm{M}_{\mathrm{sym}}^{N}\right)
\end{gathered}
$$

$$
\tilde{u} \in H^{1}\left(\Omega ; \mathbb{R}^{N}\right) \quad \text { and } \quad \tilde{U} \in L^{2}\left(\Omega ; H_{\mathrm{per}, 0}^{1}\left(Y ; \mathbb{R}^{N}\right)\right) .
$$

Clearly, $\tilde{u}=\psi(t)$ on $\partial_{D} \Omega$, so that $(\tilde{u}, \tilde{U}, P(t), Z(t)) \in \tilde{\mathcal{A}}(\psi(t))$.

We claim that the pair $(\tilde{u}, \tilde{U})$ is uniquely determined. Indeed, let $(v, V) \in H^{1}\left(\Omega ; \mathbb{R}^{N}\right) \times L^{2}\left(\Omega ; H_{\mathrm{per}, 0}^{1}\left(Y ; \mathbb{R}^{N}\right)\right)$, and $v_{n} \in H^{1}\left(\Omega ; \mathbb{R}^{N}\right)$ such that, according to Remark 3.4, $v_{n}=v-\tilde{u}$ on $\partial \Omega$,

$$
v_{n} \rightarrow v-\tilde{u} \quad \text { weakly in } H^{1}\left(\Omega ; \mathbb{R}^{N}\right)
$$

and

$$
E v_{n} \stackrel{s-2}{\rightarrow} E v-E \tilde{u}+E_{y} V-E_{y} \tilde{U} \quad \text { two-scale strongly in } L^{2}\left(\Omega \times Y ; \mathrm{M}_{\mathrm{sym}}^{N}\right) .
$$

The global stability of $\left(u_{n}(t), p_{n}(t), z_{n}(t)\right)$ yields by comparison with $\left(u_{n}(t)+v_{n}, p_{n}(t), z_{n}(t)\right) \in \mathcal{A}_{\varepsilon_{n}}(\psi(t))$

$$
\mathcal{Q}_{\varepsilon_{n}}\left(u_{n}(t), p_{n}(t), z_{n}(t)\right)-\left\langle l(t), u_{n}(t)\right\rangle \leq \mathcal{Q}_{\varepsilon_{n}}\left(u_{n}(t)+v_{n}, p_{n}(t), z_{n}(t)\right)-\left\langle l(t), u_{n}(t)+v_{n}\right\rangle .
$$

Since

$$
\begin{aligned}
\mathcal{Q}_{\varepsilon_{n}}\left(u_{n}(t)+v_{n}, p_{n}(t), z_{n}(t)\right)=\mathcal{Q}_{\varepsilon_{n}}\left(u_{n}(t), p_{n}(t), z_{n}(t)\right)+\frac{1}{2} \int_{\Omega} \mathbb{C}\left(\frac{x}{\varepsilon_{n}}\right) & E v_{n}: E v_{n} \mathrm{~d} x \\
& +\int_{\Omega} \mathbb{C}\left(\frac{x}{\varepsilon_{n}}\right) E v_{n}:\left(E u_{n}(t)-p_{n}(t)\right) \mathrm{d} x,
\end{aligned}
$$

taking the limit in (5.16) (using Prop. 3.2, points (3) and (4)) we get

$$
\begin{aligned}
0 \leq \frac{1}{2} \int_{\Omega \times Y} \mathbb{C}(y)(E v-E \tilde{u}+ & \left.E_{y} V-E_{y} \tilde{U}\right):\left(E v-E \tilde{u}+E_{y} V-E_{y} \tilde{U}\right) \mathrm{d} x \mathrm{~d} y \\
& \quad+\int_{\Omega \times Y} \mathbb{C}(y)\left(E v-E \tilde{u}+E_{y} V-E_{y} \tilde{U}\right):\left(E \tilde{u}+E_{y} \tilde{U}-P(t)\right) \mathrm{d} x \mathrm{~d} y-\langle l(t), v-\tilde{u}\rangle .
\end{aligned}
$$

Adding to both sides the quantity

$$
\frac{1}{2} \int_{\Omega \times Y} \mathbb{C}(y)\left(E \tilde{u}+E_{y} \tilde{U}-P(t)\right):\left(E \tilde{u}+E_{y} \tilde{U}-P(t)\right) \mathrm{d} x \mathrm{~d} y-\langle l(t), \tilde{u}\rangle,
$$

we deduce that the pair $(\tilde{u}, \tilde{U})$ is a minimizer (under the boundary condition for the displacement) of the map

$$
(v, V) \mapsto \frac{1}{2} \int_{\Omega \times Y} \mathbb{C}(y)\left(E v+E_{y} V\right):\left(E v+E_{y} V\right) \mathrm{d} x \mathrm{~d} y-\int_{\Omega \times Y} \mathbb{C}(y)\left(E v+E_{y} V\right): P(t) \mathrm{d} x \mathrm{~d} y-\langle l(t), v\rangle .
$$

By strict convexity we conclude that $(\tilde{u}, \tilde{U})$ is uniquely determined, so that we denote it by $(u(t), U(t))$. We infer that (without passing to a subsequence since the limit point is uniquely determined)

$$
u_{n}(t) \rightarrow u(t) \quad \text { weakly in } H^{1}\left(\Omega ; \mathbb{R}^{N}\right)
$$


and

$$
E u_{n}(t) \stackrel{w-2}{\longrightarrow} E u(t)+E_{y} U(t) \quad \text { two-scale weakly in } L^{2}\left(\Omega \times Y ; \mathrm{M}_{\mathrm{sym}}^{N}\right) .
$$

Step 3: The limit trajectory is a quasistatic evolution. Let us prove that the limit trajectory

$$
t \mapsto(u(t), U(t), P(t), Z(t)) \in \tilde{\mathcal{A}}(\psi(t))
$$

given by the previous steps satisfies the global stability and the energy balance of Definition 5.4.

Global stability follows by the same arguments of Lemma 5.6 by replacing $\left(u_{\varepsilon}^{0}, p_{\varepsilon}^{0}, z_{\varepsilon}^{0}\right)$ with $\left(u_{n}(t), p_{n}(t), z_{n}(t)\right)$, and $\left(u_{0}, U_{0}, P_{0}, Z_{0}\right)$ with $(u(t), U(t), P(t), Z(t))$.

Concerning the energy balance, let us write $\mathcal{Q}_{n}$ and $\mathcal{D}_{n}$ for $\mathcal{Q}_{\varepsilon_{n}}$ and $\mathcal{D}_{\varepsilon_{n}}$ respectively. Since

$$
\begin{aligned}
\mathcal{Q}_{n}\left(u_{n}(t), p_{n}(t), z_{n}(t)\right)= & \frac{1}{2} \int_{\Omega \times Y} \mathbb{C}(y)\left(\mathcal{T}_{\varepsilon_{n}}\left(E u_{n}(t)\right)-\mathcal{T}_{\varepsilon_{n}}\left(P_{n}(t)\right)\right):\left(\mathcal{T}_{\varepsilon_{n}}\left(E u_{n}(t)\right)-\mathcal{T}_{\varepsilon_{n}}\left(P_{n}(t)\right)\right) \\
& +\left|\mathcal{T}_{\varepsilon_{n}}\left(z_{n}(t)\right)\right|^{2} \mathrm{~d} x \mathrm{~d} y
\end{aligned}
$$

and

we obtain for every $t \in[0, T]$

$$
\mathcal{D}_{n}\left(p_{n}, z_{n} ; 0, t\right)=\tilde{\mathcal{D}}\left(\mathcal{T}_{\varepsilon_{n}}\left(p_{n}\right), \mathcal{T}_{\varepsilon_{n}}\left(z_{n}\right) ; 0, t\right),
$$

$$
\tilde{\mathcal{Q}}(u(t), U(t), P(t), Z(t)) \leq \liminf _{n \rightarrow \infty} \mathcal{Q}_{n}\left(u_{n}(t), p_{n}(t), z_{n}(t)\right)
$$

and (since $\tilde{\mathcal{D}}$ is a sort of total variation in time)

$$
\tilde{\mathcal{D}}(P, Z ; 0, t) \leq \liminf _{n \rightarrow \infty} \mathcal{D}_{n}\left(p_{n}, z_{n} ; 0, t\right) .
$$

Taking the limit for $n \rightarrow \infty$ in

$$
E_{n}(t)+\mathcal{D}_{n}\left(p_{n}, z_{n} ; 0, t\right)=E_{n}(0)-\int_{0}^{t}\left\langle\dot{l}(\tau), u_{n}(\tau)\right\rangle \mathrm{d} \tau
$$

in view of (5.9) we deduce that

$$
\tilde{E}(t)+\tilde{\mathcal{D}}(P, Z ; 0, t) \leq \lim _{n \rightarrow \infty}\left[E_{n}(t)+\mathcal{D}_{n}\left(p_{n}, z_{n} ; 0, t\right)\right]=\tilde{E}(0)-\int_{0}^{t}\langle\dot{l}(\tau), u(\tau)\rangle \mathrm{d} \tau .
$$

On the other hand, the global stability implies that for every $t \in[0, T]$ (see for example [15], Thm. 4.4)

$$
\tilde{E}(t)+\tilde{\mathcal{D}}(P, Z ; 0, t) \geq \tilde{E}(0)-\int_{0}^{t}\langle\dot{l}(\tau), u(\tau)\rangle \mathrm{d} \tau
$$

so that the energy balance condition holds. The map $t \mapsto(u(t), U(t), P(t), Z(t))$ is thus a quasistatic evolution with initial configuration $\left(u_{0}, U_{0}, P_{0}, Z_{0}\right)$. Since the evolution is uniquely determined, we conclude that the convergences (5.13)-(5.18) hold indeed along the entire family for $\varepsilon \rightarrow 0$.

Finally from (5.19) (which is indeed an equality) we infer that for every $t \in[0, T]$

$$
\lim _{\varepsilon \rightarrow 0} E_{\varepsilon}(t)=\tilde{E}(t) \quad \text { and } \quad \lim _{\varepsilon \rightarrow 0} \mathcal{D}_{\varepsilon}\left(p_{\varepsilon}, z_{\varepsilon} ; 0, t\right)=\tilde{\mathcal{D}}(P, Z ; 0, t) .
$$

This entails that for every $t \in[0, T]$

$$
E u_{\varepsilon}(t)-p_{\varepsilon}(t) \stackrel{s-2}{\longrightarrow} E u(t)+E_{y} U(t)-P(t) \quad \text { two-scale strongly in } L^{2}\left(\Omega \times Y ; \mathrm{M}_{\mathrm{sym}}^{N}\right)
$$


and

$$
z_{\varepsilon}(t) \stackrel{s-2}{\longrightarrow} Z(t) \quad \text { two-scale strongly in } L^{2}(\Omega \times Y)
$$

This completes the proof.

Remark 5.9. Reformulating the two-scale quasistatic evolution $t \mapsto(u(t), U(t), P(t), Z(t))$ in a single scale setting demands for an integration with respect to the microstructural variable $y$, so that usual weak limits $\hat{P}(t)$ and $\hat{Z}(t)$ of $p_{\varepsilon}(t)$ and $z_{\varepsilon}(t)$ are obtained (the displacement $u(t)$ is already the weak limit of $u_{\varepsilon}(t)$ ). Unfortunately, in view of the nonlinearities appearing in the global stability and the energy balance conditions, the mean with respect to $y$ cannot be performed preserving the structure of the two properties. In other words, it is likely that the evolution $t \mapsto(u(t), \hat{P}(t), \hat{Z}(t))$ cannot be interpreted as a quasistatic evolution for a homogenized standard plasticity model, although we do not have a rigorous argument supporting this conclusion. A hint regarding such a difficulty was given by the analysis of the cell problem of Fleck and Willis considered in Theorem 4.7 (see also Rem. 4.8), where the effective plastic potential in a single scale setting depends also on the elastic behaviour of the material.

The loss of information entailed by taking the mean with respect to the microstructural variable $y$ could require a description of the evolution in terms of nonlocal properties, such as memory effects, as pointed out by Tartar [20,21]. For example, in the case of linear thermoviscoelasticity Francfort and Suquet [10] showed that homogenization can induce memory effects of fading type.

Recently Visintin [22-24] dealt with the problem of formulating a single scale description for the two-scale homogenization of nonlinear problems arising in viscoelasticity and elastoplasticity. His arguments are of a variational nature, and are nonlocal in time. The case of elastoplasticity presents technical difficulties due to the linear growth of the dissipation, so that the regularity in time which can be used in the minimum problems is only that of function of bounded variation (and not Sobolev regularity as for other problems in viscoelasticity). Concerning our problem of strain gradient plasticity, Visintin's ideas amount, loosely speaking, in manipulating the energy balance by taking the minimum of the left-hand side along trajectories $t \mapsto(v(t), V(t), Q(t), \Xi(t))$ such that $\hat{v}(t)=u(t), \hat{Q}(t)=\hat{P}(t)$ and $\hat{\Xi}(t)=\hat{Z}(t)$ (the average with respect to $y$ provide the weak limits of the evolution), and which satisfy the global stability condition. Unfortunately, such a formulation seems not to provide any further physical insight into the problem.

Acknowledgements. A.G. is supported by the Italian Ministry of University and Research, project "Problemi variazionali e di evoluzione con scale multiple" 2008.

\section{REFERENCES}

[1] G. Allaire, Homogenization and two-scale convergence. SIAM J. Math. Anal. 23 (1992) 1482-1518.

[2] M.F. Ashby, The deformation of plastically non-homogeneous alloys. Philos. Mag. 21 (1970) 399-424.

[3] D. Cioranescu, A. Damlamian and G. Griso, Periodic unfolding and homogenization. C. R. Math. Acad. Sci. Paris 335 (2002) 99-104.

[4] D. Cioranescu, A. Damlamian and G. Griso, The periodic unfolding method in homogenization. SIAM J. Math. Anal. 40 (2008) 1585-1620.

[5] G. Dal Maso, A. DeSimone and M.G. Mora, Quasistatic evolution problems for linearly elastic-perfectly plastic materials. Arch. Ration. Mech. Anal. 180 (2006) 237-291.

[6] R. Dautray and J.-L. Lions, Mathematical analysis and numerical methods for science and technology 2, Functional and variational methods. Springer-Verlag, Berlin (1988).

[7] N.A. Fleck and J.W. Hutchinson, Strain gradient plasticity. Adv. Appl. Mech. 33 (1997) 295-361.

[8] N.A. Fleck and J.W. Hutchinson, A reformulation of strain gradient plasticity. J. Mech. Phys. Solids. 49 (2001) $2245-2271$.

[9] N.A. Fleck and J.R. Willis, Bounds and estimates for the effect of strain gradients upon the effective plastic properties of an isotropic two-phase composite. J. Mech. Phys. Solids 52 (2004) 1855-1888.

[10] G. Francfort and P.-M. Suquet, Homogenization and mechanical dissipation in thermoviscoelasticity. Arch. Ration. Mech. Anal. 96 (1986) 265-293.

[11] A. Giacomini and L. Lussardi, Quasi-static evolution for a model in strain gradient plasticity. SIAM J. Math. Anal. 40 (2008) 1201-1245.

[12] P. Gudmundson, A unified treatment of strain gradient plasticity. J. Mech. Phys. Solids 52 (2004) 1379-1406. 
[13] M.E. Gurtin and L. Anand, A theory of strain-gradient plasticity for isotropic, plastically irrotational materials. I. Small deformations. J. Mech. Phys. Solids 53 (2005) 1624-1649.

[14] D. Lukkassen, G. Nguetseng and P. Wall, Two-scale convergence. Int. J. Pure Appl. Math. 2 (2002) 35-86.

[15] A. Mainik and A. Mielke, Existence results for energetic models for rate-independent systems. Calc. Var. Partial Differential Equations 22 (2005) 73-99.

[16] A. Mielke, Evolution of rate-independent systems, in Handb. Differ. Equ., Evolutionary equations II, Elsevier/North-Holland, Amsterdam (2005) 461-559.

[17] A. Mielke and F. Theil, A mathematical model for rate independent phase transformations with hysteresis, in Proceedings of the Workshop on Models of Continuum Mechanics in Analysis and Engineering, H.-D. Alber, R. Balean and R. Farwig Eds., Shaker-Verlag, Aachen (1999) 117-129.

[18] A. Mielke and A.M. Timofte, Two-scale homogenization for evolutionary variational inequalities via the energetic formulation. SIAM J. Math. Anal. 39 (2007) 642-668.

[19] G. Nguetseng, A general convergence result for a functional related to the theory of homogenization. SIAM J. Math. Anal. 20 (1989) 608-623.

[20] L. Tartar, Nonlocal effects induced by homogenization, in Partial differential equations and the calculus of variations II, Progr. Nonlinear Differential Equations Appl. 2, Birkhäuser Boston, Boston (1989) 925-938.

[21] L. Tartar, Memory effects and homogenization. Arch. Ration. Mech. Anal. 111 (1990) 121-133.

[22] A. Visintin, Homogenization of the nonlinear Kelvin-Voigt model of viscoelasticity and of the Prager model of plasticity. Contin. Mech. Thermodyn. 18 (2006) 223-252.

[23] A. Visintin, Homogenization of the nonlinear Maxwell model of viscoelasticity and of the Prandtl-Reuss model of elastoplasticity. Proc. Roy. Soc. Edinburgh Sect. A 138 (2008) 1363-1401.

[24] A. Visintin, Homogenization of nonlinear visco-elastic composites. J. Math. Pures Appl. 89 (2008) 477-504.

[25] J.R. Willis, Bounds and self-consistent estimates for the overall moduli of anisotropic composites. J. Mech. Phys. Solids 25 (1977) 182-202. 